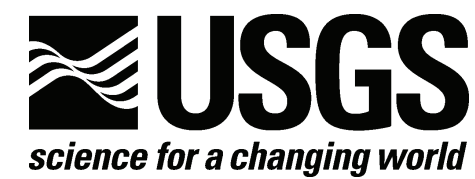

\title{
Chemical Data for Rock, Sediment, Biological, Precipitate, and Water Samples from Abandoned Copper Mines in Prince William Sound, Alaska
}

By Randolph A. Koski ${ }^{1}$ and LeeAnn Munk ${ }^{2}$, editors

${ }^{1}$ U.S. Geological Survey, Mail Stop 901, 345 Middlefield Road, Menlo Park, CA 94025

${ }^{2}$ University of Alaska-Anchorage, Department of Geological Sciences, Anchorage, AK 99508

Open-File Report 2007-1359

2007

U.S. Department of the Interior

U.S. Geological Survey 


\title{
U.S. Department of the Interior DIRK KEMPTHORNE, Secretary
}

\section{U.S. Geological Survey \\ Mark D. Myers, Director}

\author{
U.S. Geological Survey, Reston, Virginia 2007
}

For product and ordering information:

World Wide Web: http://www.usgs.gov/pubprod

Telephone: 1-888-ASK-USGS

For more information on the USGS - the Federal source for science about the Earth, its natural and living resources, natural hazards, and the environment:

World Wide Web: http://www.usgs.gov

Telephone: 1-888-ASK-USGS

Suggested citation:

Koski, R.A., and Munk, LeeAnn, eds., 2007, Chemical data for rock, sediment, biological, precipitate, and water samples from abandoned copper mines in Prince William Sound, Alaska: U.S. Geological Survey Open-File Report 2007-1359 [http://pubs.usgs.gov/of/2007/1359/].

Any use of trade, product, or firm names is for descriptive purposes only and does not imply endorsement by the U.S. Government.

Although this report is in the public domain, permission must be secured from the individual copyright owners to reproduce any copyrighted material contained within this report. 


\section{Contents}

Chapter A. Introduction

By Randolph A. Koski, LeeAnn Munk, Wayne C. Shanks, III, Lisa L. Stillings, and

Andrea L. Foster

Chapter B. Chemical data for sulfide-rich rock samples

By Randolph A. Koski......

Chapter C. Chemical data for sediment samples

By Randolph A. Koski and Wayne C. Shanks, III.

Chapter D. Chemical data for biological samples

By LeeAnn Munk and Randolph A. Koski

Chapter E. Chemical data for precipitate samples

By Andrea L. Foster. (link to Chapter E)

Chapter F. Chemical data for water samples

By Lisa L. Stillings, LeeAnn Munk, and Wayne C. Shanks (in preparation)

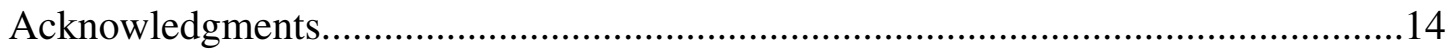

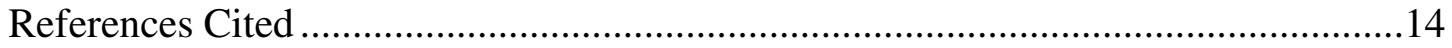

Figures

1. Location of abandoned copper mines in Prince William Sound...............................2

Tables

1. Locations and descriptions of rock samples .....................................................

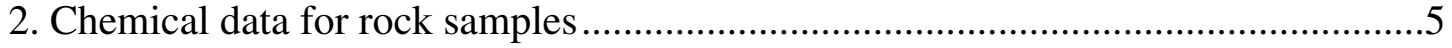

3. Locations and descriptions of sediment samples .................................................

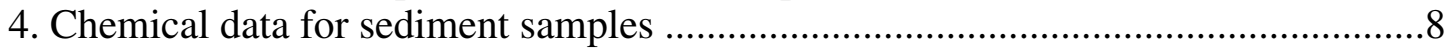

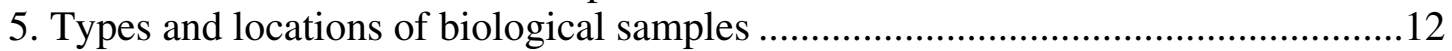

6. Chemical data (dry weight) for biological samples .........................................13

7. Locations and descriptions of precipitate samples.......................(link to Chapter E)

8. Chemical data for precipitate samples ......................................(link to Chapter E)

9. Locations and descriptions of water samples...................................(in preparation)

10. Chemical data for water samples .................................................(in preparation) 


\section{Conversion Factors}

\begin{tabular}{lll}
\hline \multicolumn{1}{c}{ Multiply } & \multicolumn{1}{c}{ By } & \multicolumn{1}{c}{ To obtain } \\
\hline Length & & \\
\hline meter (m) & 3.281 & foot (ft) \\
kilometer $(\mathrm{km})$ & 0.6214 & mile (mi) \\
\hline Volume & & \\
\hline liter (L) & 33.82 & ounce, fluid (fl. oz) \\
liter (L) & 61.02 & cubic inch (in $\left.{ }^{3}\right)$ \\
\hline Mass & & \\
\hline milligram (mg) & $3.215 \times 10^{-5}$ & ounce, troy (oz) \\
milligram (mg) & $3.527 \times 10^{-5}$ & ounce, avoirdupois (oz)
\end{tabular}

Temperature in degrees Celsius $\left({ }^{\circ} \mathrm{C}\right)$ may be converted to degrees Fahrenheit $\left({ }^{\circ} \mathrm{F}\right)$ as follows: ${ }^{\circ} \mathrm{F}=\left(1.8 x^{\circ} \mathrm{C}\right)+32$

\section{Datum}

Horizontal coordinate information is referenced to the North American Datum of 1927 (NAD 27) 


\section{Chapter A. Introduction}

By Randolph A. Koski, LeeAnn Munk, Wayne C. Shanks, III, Lisa L. Stillings, and Andrea L. Foster

In the early 20th century, approximately 6 million metric tons of copper ore were mined from numerous deposits located along the shorelines of fjords and islands in Prince William Sound, Alaska. At the Beatson, Ellamar, and Threeman mine sites (fig. 1), rocks containing $\mathrm{Fe}, \mathrm{Cu}, \mathrm{Zn}$, and $\mathrm{Pb}$ sulfide minerals are exposed to chemical weathering in abandoned mine workings and remnant waste piles that extend into the littoral zone. Field investigations in 2003 and 2005 as well as analytical data for rock, sediment, precipitate, water, and biological samples reveal that the oxidation of sulfides at these sites is resulting in the generation of acid mine drainage and the transport of metals into the marine environment (Koski and others, 2008; Stillings and others, 2008).

At the Ellamar and Threeman sites, plumes of acidic and metal-enriched water are flowing through beach gravels into the shallow offshore environment. Interstitial water samples collected from beach sediment at Ellamar have low pH levels (to $\sim 3$ ) and high concentrations of metals including iron, copper, zinc, cobalt, lead, and mercury. The abundant precipitation of the iron sulfate mineral jarosite in the Ellamar gravels also signifies a low-pH environment. At the Beatson mine site (the largest copper mine in the region) seeps containing iron-rich microbial precipitates drain into the intertidal zone below mine dumps (Foster and others, 2008). A stream flowing down to the shoreline from underground mine workings at Beatson has near-neutral $\mathrm{pH}$, but elevated levels of zinc, copper, and lead (Stillings and others, 2008). Offshore sediment samples at Beatson are enriched in these metals. Preliminary chemical data for tissue from marine mussels collected near the Ellamar, Threeman, and Beatson sites reveal elevated levels of copper, zinc, and lead compared to tissue in mussels from other locations in Prince William Sound (Koski and others, 2008).

Three papers presenting results of this ongoing investigation of sulfide oxidation in Prince William Sound are in press. Koski and others (2008) provide an overview of rock alteration, surface water chemistry, and the distribution of metals at the Ellamar, Threeman, and Beatson mine sites. Based on a 60-day, stream-discharge experiment at 
Beatson in 2005, Stillings and others (2008) analyze changes in water chemistry during storm events and the flux of metals to the shoreline. Foster and others (2008) investigate the biomass and diversity of microbial communities present in surface waters (streams, seeps, pore waters) using fatty acid methyl ester (FAMES) data and principal component analysis. The publications cited above contain a subset of the total chemical data for rock, sediment, biological, precipitate, and water samples collected from the three mine sites in 2003 and 2005. The purpose of this report is the presentation of complete chemical data sets for all samples collected during the two field periods of fieldwork. Data for a small number of samples collected at two other mines (Schlosser and Fidalgo, fig. 1), visited in 2003, are also included in the tables.

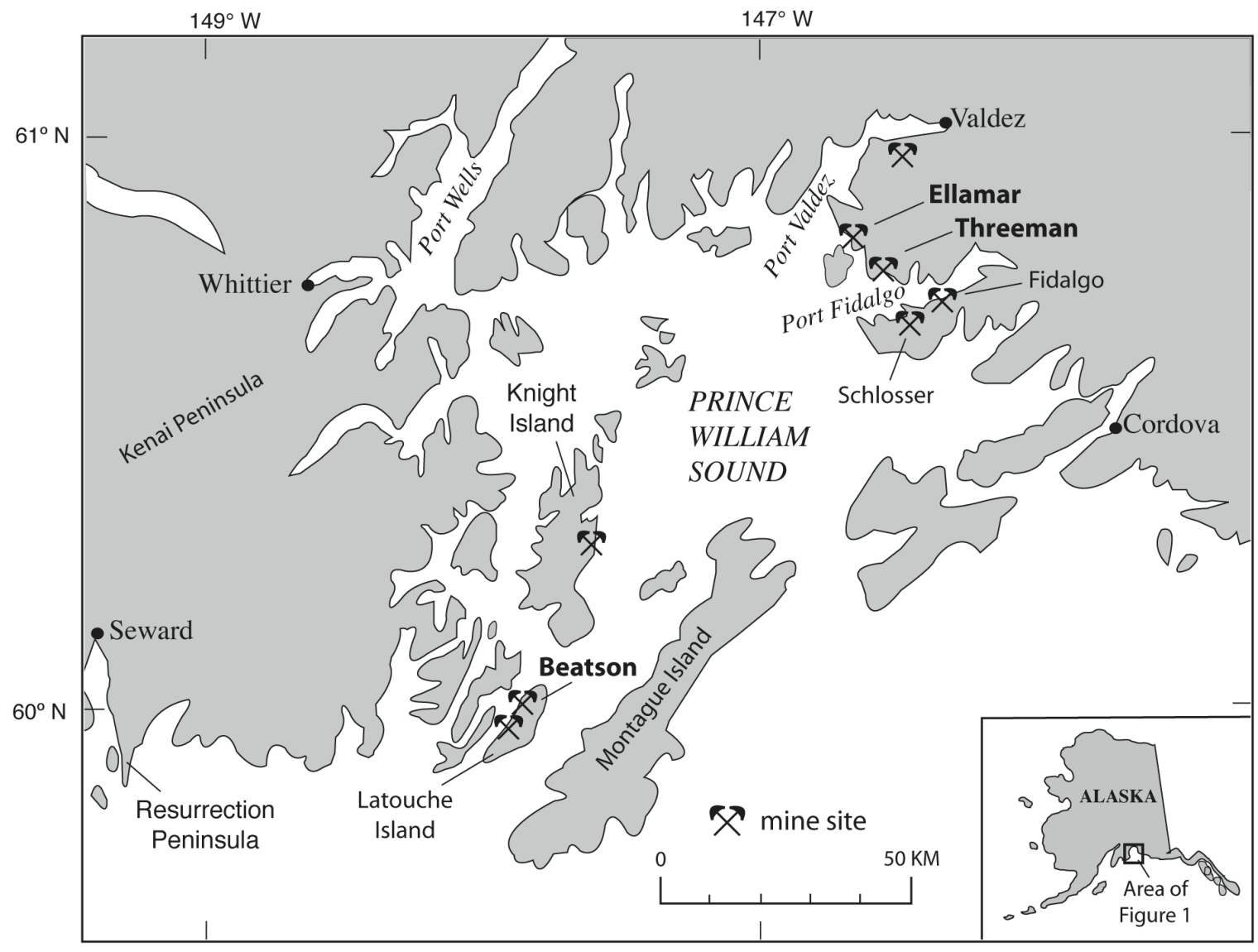

Figure 1. Location of abandoned copper mines in Prince William Sound. Chemical data for samples from named mines are presented in this report. 


\title{
Chapter B. Chemical data for sulfide-rich rock samples
}

\author{
By Randolph A. Koski
}

During fieldwork in Prince William Sound in 2003 and 2005, sulfide-bearing rock samples were collected at five mine sites from surface and underground mine workings, mine talus, ore bunkers, ore and waste dumps, and beach gravels (table1). Most samples are massive sulfide dominated by sulfide minerals including pyrrhotite, pyrite, chalcopyrite and sphalerite or semi-massive sulfide composed of sulfide minerals plus variable amounts of quartz, chlorite, talc, and carbonate minerals (Koski and others, 2008). Three of the samples (two from Threeman, one from Beatson) are deeply weathered, and contain abundant amorphous iron oxyhydroxide.

Twenty rock samples were analyzed in laboratories of the U.S. Geological Survey in Denver, Colorado (table 2). Most major and trace elements were determined by inductively coupled plasma-mass spectrometry following multi-acid $\left(\mathrm{HCl}-\mathrm{HNO}_{3}-\mathrm{HClO}_{4}-\right.$ HF) digestion (Briggs and Meier, 2002). Mercury was determined by cold-vapor atomic absorption spectroscopy (Brown et al., 2002a), Se by hydride-generation atomic absorption spectrophotometry (Hageman et al., 2002), total S and total C by combustion (Brown and Curry, 2002a,b), and $\mathrm{CO}_{2}$ and carbonate carbon by coulometric titration (Brown et al., 2002b). Additional details regarding sample preparation and detection limits are found in Taggert (2002). In addition, gold in fourteen samples was analyzed by the fire assay technique at XRAL Laboratories, Toronto, Canada. Detailed mineralogical descriptions and electron microprobe data for sulfide minerals are presented in Koski and others (2008). 
Table 1. Locations and descriptions of rock samples.

\begin{tabular}{|c|c|c|c|c|c|}
\hline Sample number & Mine site & Latitude & Longitude & Sample location & Sample description; major minerals \\
\hline PWS-03-100 & Ellamar & $60^{\circ} 53.674^{\prime}$ & $-146^{\circ} 42.082^{\prime}$ & beach gravel, intertidal & massive sulfide; pyrite \\
\hline PWS03R-1 & Ellamar & $60^{\circ} 53.674^{\prime}$ & $-146^{\circ} 42.082^{\prime}$ & beach gravel at low tide line & massive sulfide; pyrite, carbonate \\
\hline PWS03R-8A2 & Ellamar & $60^{\circ} 53.682^{\prime}$ & $-146^{\circ} 42.080^{\prime}$ & beach gravel, intertidal & massive sulfide; chalcopyrite, pyrite, carbonate \\
\hline PWS03R-8A3 & Ellamar & $60^{\circ} 53.682^{\prime}$ & $-146^{\circ} 42.080^{\prime}$ & beach gravel, intertidal & massive sulfide; pyrite, sphalerite \\
\hline PWS03R-8C & Ellamar & $60^{\circ} 53.682^{\prime}$ & $-146^{\circ} 42.080^{\prime}$ & beach gravel above high tide line & massive sulfide; pyrite, chalcopyrite, quartz \\
\hline PWS03R-9 & Ellamar & $60^{\circ} 53.696$ & $-146^{\circ} 42.120^{\prime}$ & outcrop, eastern edge of glory hole & massive pyrite; pyrite \\
\hline 05PWSE006R & Ellamar & $60^{\circ} 53.679^{\prime}$ & $-146^{\circ} 42.085^{\prime}$ & beach gravel, intertidal & massive sulfide; pyrite \\
\hline 05PWSBR001Ra & Ellamar & $60^{\circ} 53.678^{\prime}$ & $-146^{\circ} 49.304^{\prime}$ & Busby Reef ${ }^{a}$ & massive sulfide; pyrite, chalcopyrite \\
\hline PWS03R-3A & Threeman & $60^{\circ} 51.266^{\prime}$ & $-146^{\circ} 34.600^{\prime}$ & ore bunker on beach & semi-massive sulfide; chalcopyrite, pyrrhotite, quartz \\
\hline PWS03R-4A & Threeman & $60^{\circ} 51.203^{\prime}$ & $-146^{\circ} 32.281^{\prime}$ & mine talus below lowest adit & massive sulfide; pyrrhotite, chalcopyrite, quartz, chlorite \\
\hline PWS03R-5A & Threeman & $60^{\circ} 51.186^{\prime}$ & $-146^{\circ} 32.266^{\prime}$ & rubbly ore pile at base of slope & massive sulfide, oxidized; pyrrhotite chalcopyrite \\
\hline 05PWST003R & Threeman & $60^{\circ} 51.186^{\prime}$ & $-146^{\circ} 32.266^{\prime}$ & rubbly ore pile at base of slope & massive sulfide, oxidized; sulfur, chlinochlore, chalcopyrite, goethite \\
\hline 05PWSB002R & Beatson & $60^{\circ} 03.189^{\prime}$ & $-147^{\circ} 54.143^{\prime}$ & mine dump & massive sulfide; chalcopyrite; pyrrhotite \\
\hline 05PWSB004Rb & Beatson & $60^{\circ} 03.238^{\prime}$ & $-147^{\circ} 54.035^{\prime}$ & mine dump & massive sulfide; chalcopyrite, talc \\
\hline 05PWSB005R & Beatson & $60^{\circ} 03.167^{\prime}$ & $-147^{\circ} 54.078^{\prime}$ & mine dump & massive sulfide; quartz, chalcopyrite \\
\hline 05PWSB007Ra & Beatson & $60^{\circ} 02.890^{\prime}$ & $-147^{\circ} 54.250^{\prime}$ & mine dump & semi-massive sulfide; quartz, chalcopyrite, pyrite \\
\hline 05PWSB007Rb & Beatson & $60^{\circ} 02.890^{\prime}$ & $-147^{\circ} 54.250^{\prime}$ & mine dump & semi-massive sulfide; quartz, carbonate, chalcopyrite, sphalerite \\
\hline 05PWSB011R & Beatson & $60^{\circ} 02.962^{\prime}$ & $-147^{\circ} 54.272^{\prime}$ & mine dump & massive sulfide, oxidized; quartz, pyrite, chlinochlore \\
\hline PWS03R-2C & Fidalgo & $60^{\circ} 47.905^{\prime}$ & $-146^{\circ} 18.204^{\prime}$ & ore bunker on beach & semi-massive sulfide; pyrrhotite, chalcopyrite, chlinochlore \\
\hline SM-1 & Schlosser & n.r. & n.r. & mine talus below adit & massive sulfide; pyrite \\
\hline
\end{tabular}

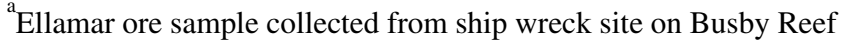

n.r. $=$ not recorded 
Table 2. Chemical data for rock samples.

\begin{tabular}{|c|c|c|c|c|c|c|c|c|c|c|c|c|}
\hline \multicolumn{3}{|c|}{ Sample numbers } & \multirow{2}{*}{$\begin{array}{l}\text { PWS-03-100 } \\
\text { Ellamar }\end{array}$} & \multirow{2}{*}{$\begin{array}{l}\text { PWS03R-1 } \\
\text { Ellamar }\end{array}$} & \multirow{2}{*}{$\begin{array}{l}\text { PWS03R-8A2 } \\
\text { Ellamar }\end{array}$} & \multirow{2}{*}{$\begin{array}{l}\text { PWS03R-8A3 } \\
\text { Ellamar }\end{array}$} & \multirow{2}{*}{$\begin{array}{l}\text { PWS03R-8C } \\
\text { Ellamar }\end{array}$} & \multirow{2}{*}{$\begin{array}{l}\text { PWS03R-9 } \\
\text { Ellamar }\end{array}$} & \multirow{2}{*}{$\begin{array}{l}\text { 05PWSE006R } \\
\text { Ellamar }\end{array}$} & \multirow{2}{*}{$\begin{array}{l}\text { 05PWSBR001Ra } \\
\text { Ellamar }\end{array}$} & \multirow{2}{*}{$\begin{array}{l}\text { PWS03R-3A } \\
\text { Threeman }\end{array}$} & \multirow{2}{*}{$\begin{array}{l}\text { PWS03R-4A } \\
\text { Threeman }\end{array}$} \\
\hline & Mine site & & & & & & & & & & & \\
\hline Element & Method & Units & & & & & & & & & & \\
\hline $\mathrm{Al}$ & ICPMS & wt. \% & 0.077 & 1.3 & 0.121 & 0.399 & 0.166 & 0.049 & 0.293 & 0.215 & 1.04 & 1.09 \\
\hline $\mathrm{CO}_{2}$ & CT & wt. \% & n.a. & n.a. & 3.42 & 3.09 & 6.72 & 0.03 & 0.01 & 0.05 & n.a. & n.a. \\
\hline CARB C & $\mathrm{CT}$ & wt. $\%$ & n.a. & n.a. & 0.93 & 0.84 & 1.83 & 0.01 & $<0.003$ & 0.01 & n.a. & n.a. \\
\hline $\mathrm{C}($ total $)$ & COMB & wt. \% & n.a. & n.a. & 1.1 & 0.97 & 1.89 & 0.1 & 0.09 & 0.09 & n.a. & n.a. \\
\hline $\mathrm{Ca}$ & ICPMS & wt. \% & 0.035 & 4.16 & 0.505 & 2.43 & 4.21 & 0.028 & 0.007 & 0.003 & 0.112 & 0.636 \\
\hline $\mathrm{Fe}$ & ICPMS & wt. \% & 38 & 23 & 26 & 28 & 30 & 42 & 41 & 24 & 33 & 20 \\
\hline K & ICPMS & wt. \% & $<0.002$ & 0.036 & $<0.002$ & 0.004 & $<0.002$ & $<0.002$ & 0.107 & $<0.002$ & 0.220 & 0.003 \\
\hline $\mathrm{Mg}$ & ICPMS & wt. \% & 0.068 & 0.362 & 0.202 & 0.205 & 0.209 & 0.0206 & 0.0146 & 0.0749 & 0.487 & 0.834 \\
\hline $\mathrm{Na}$ & ICPMS & wt. \% & 0.004 & 0.011 & 0.004 & 0.027 & 0.002 & 0.010 & 0.078 & 0.009 & 0.052 & 0.003 \\
\hline $\mathrm{P}$ & ICPMS & wt. \% & $<0.0008$ & 0.007 & $<0.0008$ & 0.001 & 0.001 & $<0.0008$ & $<0.0008$ & $<0.0008$ & 0.005 & 0.002 \\
\hline S & COMB & wt. \% & n.a. & n.a. & 34.6 & 38.2 & 35.7 & 49.9 & 52 & 34 & n.a. & n.a. \\
\hline $\mathrm{Ti}$ & ICPMS & wt. \% & 0.004 & 0.021 & $<0.004$ & 0.004 & $<0.004$ & $<0.004$ & 0.026 & 0.008 & 0.054 & 0.046 \\
\hline $\mathrm{Ag}$ & ICPMS & $\mathrm{mg} / \mathrm{kg}$ & n.a. & n.a. & 27 & 28 & 21 & 17 & 26 & 12 & n.a. & n.a. \\
\hline As & ICPMS & $\mathrm{mg} / \mathrm{kg}$ & 380 & 75 & 88 & 1000 & 120 & 320 & 280 & 250 & 800 & 98 \\
\hline $\mathrm{Au}$ & FA & $\mathrm{mg} / \mathrm{kg}$ & n.a. & n.a. & 1.17 & 20.3 & 1.48 & 2.91 & 1.8 & 1.77 & n.a. & n.a. \\
\hline $\mathrm{Ba}$ & ICPMS & $\mathrm{mg} / \mathrm{kg}$ & 24 & 272 & 13 & 156 & 57 & 13 & 54 & 2.7 & 53 & 3.7 \\
\hline $\mathrm{Be}$ & ICPMS & $\mathrm{mg} / \mathrm{kg}$ & 0.008 & 0.07 & 0.01 & 0.08 & 0.02 & 0.006 & 0.02 & 0.008 & 0.05 & 0.06 \\
\hline $\mathrm{Bi}$ & ICPMS & $\mathrm{mg} / \mathrm{kg}$ & n.a. & n.a. & 11 & 0.11 & 4.5 & 3.7 & 5.8 & 3.6 & n.a. & n.a. \\
\hline $\mathrm{Cd}$ & ICPMS & $\mathrm{mg} / \mathrm{kg}$ & 5.9 & 5.8 & 9.8 & 477 & 7.4 & 18 & 2.7 & 3.6 & 6.4 & 17 \\
\hline $\mathrm{Ce}$ & ICPMS & $\mathrm{mg} / \mathrm{kg}$ & $<0.05$ & 10 & $<0.5$ & 0.84 & 2.3 & $<0.5$ & $<0.5$ & 2.4 & 3 & 2.6 \\
\hline Co & ICPMS & $\mathrm{mg} / \mathrm{kg}$ & 25.5 & 416 & 786 & 7.7 & 435 & 62 & 14 & 529 & 854 & 323 \\
\hline $\mathrm{Cr}$ & ICPMS & $\mathrm{mg} / \mathrm{kg}$ & 3.4 & 17 & 1.6 & 1.6 & 0.8 & 0.4 & 2.8 & 2.6 & 34 & 47 \\
\hline Cs & ICPMS & $\mathrm{mg} / \mathrm{kg}$ & 0.02 & 0.11 & 0.05 & 0.03 & 0.03 & 0.02 & 0.06 & 0.02 & 0.11 & 0.02 \\
\hline $\mathrm{Cu}$ & ICPMS & $\mathrm{mg} / \mathrm{kg}$ & 7760 & 67200 & 122000 & 2010 & 58200 & 3140 & 1360 & 150000 & 23600 & 104000 \\
\hline $\mathrm{Ga}$ & ICPMS & $\mathrm{mg} / \mathrm{kg}$ & 1.6 & 6.1 & 6.4 & 120 & 1.8 & 3.6 & 2.3 & 2 & 3.7 & 3.3 \\
\hline $\mathrm{Hg}$ & CVAA & $\mathrm{mg} / \mathrm{kg}$ & n.a. & n.a. & 0.64 & 61 & 0.05 & 4.4 & 7.2 & 0.48 & n.a. & n.a. \\
\hline $\mathrm{La}$ & ICPMS & $\mathrm{mg} / \mathrm{kg}$ & $<0.3$ & 5.1 & $<0.3$ & 0.71 & 1.7 & $<0.3$ & $<0.3$ & 1.2 & 1.3 & 1.4 \\
\hline $\mathrm{Li}$ & ICPMS & $\mathrm{mg} / \mathrm{kg}$ & $<0.2$ & 38 & $<0.2$ & 1.2 & $<0.2$ & $<0.2$ & $<0.2$ & $<0.2$ & 2.5 & $<0.2$ \\
\hline $\mathrm{Mn}$ & ICPMS & $\mathrm{mg} / \mathrm{kg}$ & 68 & 638 & 334 & 359 & 453 & 255 & 6.3 & 14 & 228 & 337 \\
\hline Мо & ICPMS & $\mathrm{mg} / \mathrm{kg}$ & 14 & 4.4 & 13 & 18 & 15 & 16 & 16 & 0.92 & 1.9 & 0.97 \\
\hline $\mathrm{Nb}$ & ICPMS & $\mathrm{mg} / \mathrm{kg}$ & $<2$ & $<2$ & $<2$ & $<2$ & $<2$ & $<2$ & $<2$ & $<2$ & $<2$ & $<2$ \\
\hline $\mathrm{Ni}$ & ICPMS & $\mathrm{mg} / \mathrm{kg}$ & 8.2 & 29 & 1.5 & 4.8 & 2.6 & 1.7 & 4.6 & 3.4 & 82 & 130 \\
\hline $\mathrm{Pb}$ & ICPMS & $\mathrm{mg} / \mathrm{kg}$ & 93 & 23 & 23 & 325 & 42 & 185 & 258 & 32 & 1570 & 15 \\
\hline $\mathrm{Rb}$ & ICPMS & $\mathrm{mg} / \mathrm{kg}$ & 0.06 & 1.5 & 0.05 & 0.11 & 0.03 & 0.06 & 3.2 & 0.05 & 6.6 & 0.2 \\
\hline $\mathrm{Sb}$ & ICPMS & $\mathrm{mg} / \mathrm{kg}$ & 9.7 & 1.4 & 1.6 & 23 & 4.2 & 16 & 6.4 & 7.7 & 1.4 & 0.05 \\
\hline $\mathrm{Sc}$ & ICPMS & $\mathrm{mg} / \mathrm{kg}$ & 0.4 & 2.4 & $<0.3$ & 0.3 & $<0.3$ & $<0.3$ & 0.6 & 0.5 & 4.8 & 4.8 \\
\hline $\mathrm{Se}$ & HYD & $\mathrm{mg} / \mathrm{kg}$ & n.a. & n.a. & 200 & 7.2 & 170 & 17 & 140 & 440 & n.a. & n.a. \\
\hline $\mathrm{Sr}$ & ICPMS & $\mathrm{mg} / \mathrm{kg}$ & 2.3 & 39 & 4.3 & 24 & 30 & 1.1 & 2.3 & 4.6 & 6 & 11 \\
\hline Ta & ICPMS & $\mathrm{mg} / \mathrm{kg}$ & n.a. & n.a. & $<0.2$ & $<0.2$ & $<0.2$ & $<0.2$ & $<0.2$ & $<0.2$ & n.a. & n.a. \\
\hline Th & ICPMS & $\mathrm{mg} / \mathrm{kg}$ & 0.03 & 1.9 & 0.06 & 0.08 & 0.06 & $<0.03$ & 0.38 & 0.49 & 0.59 & 0.28 \\
\hline $\mathrm{Tl}$ & ICPMS & $\mathrm{mg} / \mathrm{kg}$ & 22 & 2.6 & 2.4 & 17 & 3.9 & 8.5 & 0.59 & 1.9 & $<0.003$ & $<0.003$ \\
\hline U & ICPMS & $\mathrm{mg} / \mathrm{kg}$ & 0.26 & 2.1 & 4.6 & 4.9 & 2.8 & 1.7 & 0.97 & 0.16 & 0.16 & 0.08 \\
\hline V & ICPMS & $\mathrm{mg} / \mathrm{kg}$ & 5.3 & 27 & 7.4 & 42 & 4.6 & 8.8 & 9.3 & 4.5 & 37 & 29 \\
\hline Y & ICPMS & $\mathrm{mg} / \mathrm{kg}$ & $<0.3$ & 4.9 & $<0.3$ & 0.58 & 0.94 & $<0.3$ & $<0.3$ & $<0.3$ & 3 & 1.1 \\
\hline $\mathrm{Zn}$ & ICPMS & $\mathrm{mg} / \mathrm{kg}$ & 1880 & 1800 & 3740 & 169000 & 2460 & 7700 & 679 & 1030 & 2400 & 4760 \\
\hline
\end{tabular}

ICPMS = Inductively coupled plasma-mass spectrometry; CT = Coulometric titration; COMB = Combustion in Leco analyzer; FA = Fire assay; CVAA = Cold vapor-atomic absorption spectrometry; HYD =

Hydride generation-atomic absorption spectrophotometry; CARB C = carbonate carbon; n.a. = not analyzed 
Table 2. (cont.)

\begin{tabular}{|c|c|c|c|c|c|c|c|c|c|c|c|c|}
\hline \multicolumn{3}{|c|}{ Sample numbers } & \multirow{3}{*}{$\begin{array}{l}\text { PWS03R-5A } \\
\text { Threeman }\end{array}$} & \multirow{2}{*}{$\begin{array}{l}\text { 05PWST003R } \\
\text { Threeman }\end{array}$} & \multirow{2}{*}{$\begin{array}{l}\text { 05PWSB002R } \\
\text { Beatson }\end{array}$} & \multirow{2}{*}{$\begin{array}{l}\text { 05PWSB004Rb } \\
\text { Beatson }\end{array}$} & \multirow{2}{*}{$\begin{array}{l}\text { 05PWSB005R } \\
\text { Beatson }\end{array}$} & \multirow{2}{*}{$\begin{array}{l}\text { 05PWSB007Ra } \\
\text { Beatson }\end{array}$} & \multirow{2}{*}{$\begin{array}{l}\text { 05PWSB007Rb } \\
\text { Beatson }\end{array}$} & \multirow{2}{*}{$\begin{array}{l}\text { 05PWSB011R } \\
\text { Beatson }\end{array}$} & \multirow{2}{*}{$\begin{array}{l}\text { PWS03R-2C } \\
\text { Fidalgo }\end{array}$} & \multirow{2}{*}{$\begin{array}{l}\text { SM-1 } \\
\text { Schlosse }\end{array}$} \\
\hline & Mine site & & & & & & & & & & & \\
\hline Element & Method & Units & & & & & & & & & & \\
\hline Al & ICPMS & wt. \% & 0.900 & 1.18 & 0.083 & 1.8 & 0.436 & 0.828 & 0.20 & 5.69 & 1.39 & 0.016 \\
\hline $\mathrm{CO}_{2}$ & CT & wt. \% & $<0.01$ & 0.04 & 0.17 & 0.02 & 0.13 & $<0.01$ & 4.64 & 1.08 & n.a. & n.a. \\
\hline CARB C & $\mathrm{CT}$ & wt. $\%$ & $<0.003$ & 0.01 & 0.05 & 0.01 & 0.04 & $<0.003$ & 1.27 & 0.29 & n.a. & n.a. \\
\hline C(total) & COMB & wt. \% & $<0.01$ & 0.67 & 0.06 & 0.05 & 0.03 & 0.03 & 1.27 & 0.46 & n.a. & n.a. \\
\hline $\mathrm{Ca}$ & ICPMS & wt. \% & 0.078 & 0.213 & 0.068 & 0.054 & 0.064 & 0.036 & 1.86 & 0.799 & 0.060 & 0.005 \\
\hline $\mathrm{Fe}$ & ICPMS & wt. \% & 44 & 40 & 22 & 21 & 16 & 19 & 23 & 14 & 26 & 38 \\
\hline K & ICPMS & wt. \% & 0.003 & 0.060 & $<0.002$ & $<0.002$ & $<0.002$ & $<0.002$ & 0.003 & 0.043 & 0.113 & $<0.002$ \\
\hline $\mathrm{Mg}$ & ICPMS & wt. \% & 0.0625 & 0.578 & 0.106 & 4.53 & 0.516 & 1.18 & 0.987 & 8.97 & 1.04 & 0.0060 \\
\hline $\mathrm{Na}$ & ICPMS & wt. $\%$ & 0.010 & 0.107 & 0.006 & 0.003 & 0.004 & 0.003 & 0.006 & 0.007 & 0.058 & 0.002 \\
\hline $\mathrm{P}$ & ICPMS & wt. \% & $<0.0008$ & 0.002 & $<0.0008$ & 0.038 & 0.011 & 0.016 & $<0.0008$ & 0.045 & 0.008 & 0.001 \\
\hline $\mathrm{S}$ & COMB & wt. $\%$ & 35.3 & 14.3 & 32.2 & 19.7 & 22.3 & 29.8 & 24 & 3.59 & n.a. & n.a. \\
\hline $\mathrm{Ti}$ & ICPMS & wt. \% & $<0.004$ & 0.064 & $<0.004$ & 0.006 & 0.005 & 0.005 & $<0.004$ & 0.063 & 0.055 & $<0.0002$ \\
\hline $\mathrm{Ag}$ & ICPMS & $\mathrm{mg} / \mathrm{kg}$ & 7.5 & 4.8 & 54 & 45 & 64 & 64 & 47 & 7.7 & n.a. & n.a. \\
\hline As & ICPMS & $\mathrm{mg} / \mathrm{kg}$ & 8.7 & 13 & 22 & 24 & 9.5 & 340 & 9100 & 66 & 17 & 160 \\
\hline $\mathrm{Au}$ & FA & $\mathrm{mg} / \mathrm{kg}$ & 0.042 & 0.013 & 0.082 & 0.069 & 0.066 & 0.157 & 1.61 & 0.015 & n.a. & n.a. \\
\hline $\mathrm{Ba}$ & ICPMS & $\mathrm{mg} / \mathrm{kg}$ & $<0.5$ & 13 & 9.8 & 14 & 8.6 & 9.8 & 66 & 147 & 71 & 9.1 \\
\hline $\mathrm{Be}$ & ICPMS & $\mathrm{mg} / \mathrm{kg}$ & 0.004 & 0.03 & 0.009 & 0.08 & 0.04 & 0.04 & 0.03 & 0.22 & 0.06 & 0.02 \\
\hline $\mathrm{Bi}$ & ICPMS & $\mathrm{mg} / \mathrm{kg}$ & 2.7 & 1.9 & 12 & 45 & 21 & 16 & 59 & 3.7 & n.a. & n.a. \\
\hline $\mathrm{Cd}$ & ICPMS & $\mathrm{mg} / \mathrm{kg}$ & 5.1 & 1.8 & 24 & 13 & 7.3 & 14 & 153 & 3.1 & 4.5 & 1.5 \\
\hline $\mathrm{Ce}$ & ICPMS & $\mathrm{mg} / \mathrm{kg}$ & $<0.5$ & 0.95 & $<0.5$ & 0.84 & 0.8 & 1.5 & 1.9 & 29 & 2.2 & 1.1 \\
\hline Co & ICPMS & $\mathrm{mg} / \mathrm{kg}$ & 580 & 97 & 143 & 114 & 52 & 268 & 70 & 18 & 109 & 31 \\
\hline $\mathrm{Cr}$ & ICPMS & $\mathrm{mg} / \mathrm{kg}$ & 0.3 & 49 & $<0.2$ & 5.9 & 2.2 & 4.5 & 1.2 & 69 & 11 & $<0.2$ \\
\hline Cs & ICPMS & $\mathrm{mg} / \mathrm{kg}$ & 0.01 & 0.06 & 0.02 & 0.04 & 0.03 & 0.03 & 0.07 & 0.25 & 0.20 & 0.008 \\
\hline $\mathrm{Cu}$ & ICPMS & $\mathrm{mg} / \mathrm{kg}$ & 36200 & 26700 & 155000 & 95600 & 150000 & 106000 & 73600 & 10400 & 84000 & 2020 \\
\hline $\mathrm{Ga}$ & ICPMS & $\mathrm{mg} / \mathrm{kg}$ & 0.45 & 2.9 & 1.6 & 14 & 2.6 & 3.9 & 2.8 & 15 & 6.9 & 0.28 \\
\hline $\mathrm{Hg}$ & CVAA & $\mathrm{mg} / \mathrm{kg}$ & 0.04 & 0.04 & 0.06 & 0.02 & $<0.02$ & $<0.02$ & $<0.02$ & 2.9 & n.a. & n.a. \\
\hline $\mathrm{La}$ & ICPMS & $\mathrm{mg} / \mathrm{kg}$ & $<0.3$ & 0.45 & $<0.3$ & 0.41 & 0.41 & 0.6 & 1.1 & 16 & 1.2 & 0.56 \\
\hline $\mathrm{Li}$ & ICPMS & $\mathrm{mg} / \mathrm{kg}$ & $<0.2$ & $<0.2$ & $<0.2$ & 1.3 & 2.1 & 0.2 & $<0.2$ & 7.5 & 1.6 & $<0.2$ \\
\hline $\mathrm{Mn}$ & ICPMS & $\mathrm{mg} / \mathrm{kg}$ & 73 & 380 & 132 & 184 & 128 & 114 & 1350 & 1450 & 344 & 11 \\
\hline Мo & ICPMS & $\mathrm{mg} / \mathrm{kg}$ & 1.8 & 19 & 0.93 & 11 & 1.2 & 0.68 & 2.1 & 0.67 & 11 & 6.3 \\
\hline $\mathrm{Nb}$ & ICPMS & $\mathrm{mg} / \mathrm{kg}$ & $<2$ & $<2$ & $<2$ & $<2$ & $<2$ & $<2$ & $<2$ & $<2$ & $<2$ & $<2$ \\
\hline $\mathrm{Ni}$ & ICPMS & $\mathrm{mg} / \mathrm{kg}$ & 129 & 19 & 45 & 5 & 17 & 12 & 17 & 12 & 15 & $<1$ \\
\hline $\mathrm{Pb}$ & ICPMS & $\mathrm{mg} / \mathrm{kg}$ & 5.1 & 4.7 & 27 & 52 & 404 & 559 & 4150 & 69 & 22 & 53 \\
\hline $\mathrm{Rb}$ & ICPMS & $\mathrm{mg} / \mathrm{kg}$ & 0.28 & 1.1 & 0.11 & 0.07 & 0.07 & 0.06 & 0.11 & 1.3 & 4.2 & 0.08 \\
\hline $\mathrm{Sb}$ & ICPMS & $\mathrm{mg} / \mathrm{kg}$ & 0.08 & 0.42 & 4.3 & 1.9 & 3.1 & 7.1 & 134 & 2.1 & $<0.02$ & 5.6 \\
\hline $\mathrm{Sc}$ & ICPMS & $\mathrm{mg} / \mathrm{kg}$ & $<0.3$ & 4.5 & $<0.3$ & 1.1 & 0.5 & 0.8 & 0.6 & 9 & 2.1 & $<0.3$ \\
\hline $\mathrm{Se}$ & HYD & $\mathrm{mg} / \mathrm{kg}$ & 380 & 170 & 200 & 210 & 280 & 210 & 78 & 16 & n.a. & n.a. \\
\hline $\mathrm{Sr}$ & ICPMS & $\mathrm{mg} / \mathrm{kg}$ & 1.5 & 11 & 5.1 & 4.1 & 2.4 & 1.8 & 128 & 109 & 3.8 & 1.4 \\
\hline Тa & ICPMS & $\mathrm{mg} / \mathrm{kg}$ & $<0.2$ & $<0.2$ & $<0.2$ & 0.28 & $<0.2$ & $<0.2$ & $<0.2$ & 0.33 & n.a. & n.a. \\
\hline Th & ICPMS & $\mathrm{mg} / \mathrm{kg}$ & $<0.03$ & 0.16 & 0.04 & 0.35 & 0.33 & 0.33 & 0.1 & 4.7 & 1.2 & 0.1 \\
\hline $\mathrm{Tl}$ & ICPMS & $\mathrm{mg} / \mathrm{kg}$ & 0.02 & 0.03 & 0.2 & 0.09 & 0.2 & 0.71 & 0.75 & 0.07 & $<0.003$ & 5.6 \\
\hline $\mathrm{U}$ & ICPMS & $\mathrm{mg} / \mathrm{kg}$ & 0.05 & 1.8 & 0.04 & 1.3 & 0.13 & 0.14 & 0.04 & 0.71 & 0.48 & 0.25 \\
\hline V & ICPMS & $\mathrm{mg} / \mathrm{kg}$ & 1.3 & 39 & 1.1 & 21 & 4 & 11 & 1.7 & 86 & 29 & $<0.4$ \\
\hline Y & ICPMS & $\mathrm{mg} / \mathrm{kg}$ & $<0.3$ & 2.2 & $<0.3$ & 0.96 & 0.46 & 0.54 & 3.1 & 3.6 & 1.1 & 2.6 \\
\hline $\mathrm{Zn}$ & ICPMS & $\mathrm{mg} / \mathrm{kg}$ & 1320 & 706 & 7690 & 3500 & 2390 & 4760 & 57000 & 1170 & 942 & 511 \\
\hline
\end{tabular}




\title{
Chapter C. Chemical data for sediment samples
}

\author{
By Randolph A. Koski and Wayne C. Shanks, III
}

During studies of sulfide oxidation in coastal areas of Prince William Sound in 2003 and 2005, sediment samples were collected from the intertidal zones and in offshore locations near several abandoned mine sites (table 3). Intertidal sediment samples from the beach environment range from fine to coarse sand whereas offshore samples are mixtures of mud, silt, and fine sand. The offshore samples were collected by dredging and coring devices deployed from a small boat as well as by remotely operated vehicle. Twenty-six sediment samples from the Ellamar, Threeman, and Beatson sites were analyzed in laboratories of the U.S. Geological Survey in Denver, Colorado (table 4). Major and trace elements were determined by inductively coupled plasma-mass spectrometry following multi-acid $\left(\mathrm{HCl}-\mathrm{HNO}_{3}-\mathrm{HClO}_{4}-\mathrm{HF}\right)$ digestion (Briggs and Meier, 2002) except for mercury, which was analyzed by cold-vapor atomic absorption spectroscopy (Brown et al., 2002). Additional details regarding sample preparation and detection limits are found in Taggert (2002). A discussion of metal concentrations in the sedimentary deposits is presented in Koski and others (2008).

Table 3. Locations and descriptions of sediment samples.

\begin{tabular}{|c|c|c|c|c|c|}
\hline Sample Number & Mine site & Latitude & Longitude & Sample Location & Sample Description \\
\hline 03Ellamar-Pit 1 & Ellamar & $60^{\circ} 53.682^{\prime}$ & $-146^{\circ} 42.083^{\prime}$ & intertidal zone near Ellamar pier, beach pit & sand with jarosite precipitate \\
\hline 03Ellamar-Pit 2 & Ellamar & $60^{\circ} 53.682^{\prime}$ & $-146^{\circ} 42.083^{\prime}$ & intertidal zone near Ellamar pier, beach pit & sand with jarosite precipitate \\
\hline 03Ellamar-Pit 3 & Ellamar & $60^{\circ} 53.682^{\prime}$ & $-146^{\circ} 42.083^{\prime}$ & intertidal zone near Ellamar pier, beach pit & sand with jarosite precipitate \\
\hline 03Ellamar-Pit 4 & Ellamar & $60^{\circ} 53.682^{\prime}$ & $-146^{\circ} 42.083^{\prime}$ & intertidal zone near Ellamar pier, beach pit & sand with jarosite precipitate \\
\hline 03Ellamar-Pit 5 & Ellamar & $60^{\circ} 53.682^{\prime}$ & $-146^{\circ} 42.083^{\prime}$ & intertidal zone near Ellamar pier, beach pit & sand with jarosite precipitate \\
\hline PWS03-SELL-4 & Ellamar & & n.r. & shallow offshore near end of Ellamar pier & sand \\
\hline PWS03S-3 & Ellamar & $60^{\circ} 53.696^{\prime}$ & $-146^{\circ} 42.134^{\prime}$ & offshore, bottom of Ellamar glory hole & silt \\
\hline PWS030S-102 & Ellamar & n.r. & n.r. & offshore & mud \\
\hline 05PWSE-002S & Ellamar & $60^{\circ} 53.662^{\prime}$ & $-146^{\circ} 41.998^{\prime}$ & upper intertidal zone & red beach sand \\
\hline 05PWSE-008S & Ellamar & $60^{\circ} 53.637^{\prime}$ & $-146^{\circ} 42.156^{\prime}$ & offshore, $6 \mathrm{~m}$ water depth & olive brown silt and sand \\
\hline 05PWSE-108S & Ellamar & $60^{\circ} 53.701^{\prime}$ & $-146^{\circ} 42.239^{\prime}$ & shallow offshore, near Ellamar glory hole & brownish-black sand \\
\hline 05PWSE-110R & Ellamar & $60^{\circ} 53.653^{\prime}$ & $-146^{\circ} 42.093^{\prime}$ & offshore, near low tide line & dark gray sand \\
\hline 05PWSE-111S & Ellamar & $60^{\circ} 53.653^{\prime}$ & $-146^{\circ} 42.093^{\prime}$ & offshore, near low tide line & black mud and silt \\
\hline 05PWSE-112S & Ellamar & $60^{\circ} 53.582^{\prime}$ & $-146^{\circ} 42.355^{\prime}$ & offshore, $5 \mathrm{~m}$ water depth & gray sand \\
\hline 05PWSE-207S & Ellamar & $60^{\circ} 53.680^{\prime}$ & $-146^{\circ} 42.086^{\prime}$ & intertidal zone near Ellamar pier, beach pit & brown sand \\
\hline 05PWSE-208S & Ellamar & $60^{\circ} 53.680^{\prime}$ & $-146^{\circ} 42.086^{\prime}$ & intertidal zone near Ellamar pier, beach pit & brown sand \\
\hline 05PWST-115S & Threeman & $60^{\circ} 51.068^{\prime}$ & $-146^{\circ} 32.124^{\prime}$ & offshore, $14 \mathrm{~m}$ water depth & olive-brown silt \\
\hline 05PWST-118S & Threeman & $60^{\circ} 51.068^{\prime}$ & $-146^{\circ} 32.243^{\prime}$ & lower intertidal zone & sand with $\mathrm{Fe}$-oxyhydroxide precipitate \\
\hline 05PWST-120S & Threeman & $60^{\circ} 51.150^{\prime}$ & $-146^{\circ} 32.247^{\prime}$ & offshore, $3 \mathrm{~m}$ water depth & olive-brown silt \\
\hline 05PWST-304S-1 & Threeman & $60^{\circ} 51.206^{\prime}$ & $-146^{\circ} 32.228^{\prime}$ & intertidal zone, beach pit & gray sand \\
\hline 05PWST-305SM-1 & Threeman & $60^{\circ} 51.180^{\prime}$ & $-146^{\circ} 32.260^{\prime}$ & intertidal zone, beach pit & gray sand \\
\hline 05PWST-306SM-2 & Threeman & $60^{\circ} 51.182^{\prime}$ & $-146^{\circ} 32.261^{\prime}$ & intertidal zone, beach pit & olive-brown sand \\
\hline 05PWST-308SM-1 & Threeman & & n.r. & upper intertidal zone & beach sand near ore pile \\
\hline 05PWSB-010S & Beatson & $60^{\circ} 03.287^{\prime}$ & $-147^{\circ} 54.344^{\prime}$ & offshore, $33 \mathrm{~m}$ water depth & gray-brown mud and silt \\
\hline 05PWSB-101S & Beatson & $60^{\circ} 03.209^{\prime}$ & $-147^{\circ} 54.201^{\prime}$ & offshore, near low tide line & sand \\
\hline 05PWSB-104S & Beatson & $60^{\circ} 03.213^{\prime}$ & $-147^{\circ} 54.272^{\prime}$ & offshore, low tide line & sand \\
\hline
\end{tabular}
n.r. $=$ not recorded 
Table 4. Chemical data for sediment samples.

\begin{tabular}{|c|c|c|c|c|c|c|c|c|c|c|c|c|c|c|c|}
\hline \multicolumn{3}{|c|}{ Sample numbers } & $\begin{array}{l}\text { 03Ellamar- } \\
\text { Pit } 5\end{array}$ & $\begin{array}{l}\text { 03Ellamar- } \\
\text { Pit } 4\end{array}$ & $\begin{array}{l}\text { 03Ellamar- } \\
\text { Pit } 2\end{array}$ & $\begin{array}{l}\text { 03Ellamar- } \\
\text { Pit } 1\end{array}$ & $\begin{array}{l}\text { 03Ellamar- } \\
\text { Pit } 3\end{array}$ & $\begin{array}{l}\text { PWS03- } \\
\text { SELL-4 }\end{array}$ & $\begin{array}{l}\text { PWS03S- } \\
3\end{array}$ & $\begin{array}{l}\text { PWS030S- } \\
102\end{array}$ & $\begin{array}{l}\text { 05PWSE- } \\
002 \mathrm{~S}\end{array}$ & $\begin{array}{l}\text { 05PWSE- } \\
008 \mathrm{~S}\end{array}$ & $\begin{array}{l}\text { 05PWSE- } \\
108 \mathrm{~S}\end{array}$ & $\begin{array}{l}\text { 05PWSE- } \\
110 \mathrm{R}\end{array}$ & $\begin{array}{l}\text { 05PWSE- } \\
111 \mathrm{~S}\end{array}$ \\
\hline \multicolumn{3}{|c|}{ Mine site } & Ellamar & Ellamar & Ellamar & Ellamar & Ellamar & Ellamar & Ellamar & Ellamar & Ellamar & Ellamar & Ellamar & Ellamar & Ellamar \\
\hline \multicolumn{3}{|c|}{ Location } & intertidal & intertidal & intertidal & intertidal & intertidal & offshore & $\begin{array}{l}\text { glory } \\
\text { hole }\end{array}$ & offshore & intertidal & offshore & offshore & offshore & offshore \\
\hline Element & Method & Units & & & & & & & & & & & & & \\
\hline $\mathrm{Al}$ & ICPMS & wt. $\%$ & 2.99 & 0.65 & 3.65 & 4.19 & 5.37 & 5.84 & 7.06 & 6.12 & 4.75 & 6.56 & 4.39 & 7.24 & 6.58 \\
\hline $\mathrm{Ca}$ & ICPMS & wt. $\%$ & 0.061 & 0.028 & 0.046 & 0.042 & 0.059 & 3.66 & 0.494 & 1.87 & 0.124 & 1.32 & 2.23 & 1.47 & 1.64 \\
\hline $\mathrm{Fe}$ & ICPMS & wt. $\%$ & 18.8 & 40.6 & 24.5 & 18.6 & 19.3 & 5.86 & 9.95 & 4.65 & 19 & 3.4 & 4.5 & 4.5 & 5.1 \\
\hline K & ICPMS & wt. $\%$ & 1.27 & 2.02 & 2.43 & 2.8 & 2.73 & 1.07 & 1.53 & 1.14 & 1.60 & 1.28 & 1.11 & 1.54 & 1.36 \\
\hline $\mathrm{Mg}$ & ICPMS & wt. $\%$ & 0.281 & 0.0713 & 0.148 & 0.0888 & 0.512 & 1.61 & 1.24 & 1.64 & 0.64 & 1.42 & 1.64 & 1.61 & 1.47 \\
\hline $\mathrm{Na}$ & ICPMS & wt. $\%$ & 0.624 & 0.206 & 0.695 & 1.8 & 0.786 & 3.05 & 1.87 & 3.48 & 0.746 & 1.65 & 4.32 & 1.73 & 1.64 \\
\hline $\mathrm{P}$ & ICPMS & wt. $\%$ & 0.0514 & 0.0284 & 0.023 & 0.0177 & 0.0808 & 0.174 & 0.152 & 0.116 & 0.057 & 0.066 & 0.20 & 0.10 & 0.17 \\
\hline $\mathrm{Ti}$ & ICPMS & wt. $\%$ & 0.219 & 0.0874 & 0.253 & 0.256 & 0.311 & 0.456 & 0.451 & 0.466 & 0.21 & 0.37 & 0.24 & 0.42 & 0.38 \\
\hline $\mathrm{Ag}$ & ICPMS & $\mathrm{mg} / \mathrm{kg}$ & 62 & 5.5 & 12 & 1.0 & 2.5 & 0.50 & 0.70 & 0.34 & 3.5 & $<3$ & $<3$ & $<3$ & $<3$ \\
\hline As & ICPMS & $\mathrm{mg} / \mathrm{kg}$ & 284 & 346 & 196 & 43 & 191 & 23 & 64 & 16 & 160 & 7.1 & 34 & 17 & 31 \\
\hline $\mathrm{Au}$ & ICPMS & $\mathrm{mg} / \mathrm{kg}$ & 9.9 & 1.8 & 0.60 & $<0.2$ & $<0.2$ & $<0.2$ & $<0.2$ & $<0.2$ & n.a. & n.a. & n.a. & n.a. & n.a. \\
\hline $\mathrm{Ba}$ & ICPMS & $\mathrm{mg} / \mathrm{kg}$ & 76 & 150 & 96 & 224 & 635 & 500 & 865 & 528 & 466 & 590 & 410 & 743 & 635 \\
\hline $\mathrm{Be}$ & ICPMS & $\mathrm{mg} / \mathrm{kg}$ & 0.76 & 0.12 & 0.72 & 0.64 & 1.0 & 0.88 & 1.3 & 0.9 & 0.86 & 1.1 & 0.81 & 1.3 & 1.2 \\
\hline $\mathrm{Bi}$ & ICPMS & $\mathrm{mg} / \mathrm{kg}$ & 15 & 3.0 & 5.1 & 2.6 & 2 & 0.3 & 1.1 & 0.23 & 1.8 & 0.090 & 0.34 & 0.20 & 0.36 \\
\hline $\mathrm{Cd}$ & ICPMS & $\mathrm{mg} / \mathrm{kg}$ & 1.4 & 0.38 & 3.4 & 0.031 & 0.06 & $<0.01$ & 0.70 & 0.78 & 0.30 & 0.33 & 0.43 & 0.13 & 0.45 \\
\hline $\mathrm{Ce}$ & ICPMS & $\mathrm{mg} / \mathrm{kg}$ & 14 & 4.0 & 22 & 17 & 29 & 26 & 35 & 29 & 23 & 30 & 22 & 33 & 33 \\
\hline Co & ICPMS & $\mathrm{mg} / \mathrm{kg}$ & 50 & 68 & 14 & 11 & 7.5 & 14 & 22 & 11 & 32 & 8.0 & 8.9 & 12 & 13 \\
\hline $\mathrm{Cr}$ & ICPMS & $\mathrm{mg} / \mathrm{kg}$ & 38 & 9.8 & 35 & 38 & 66 & 81 & 91 & 80 & 70 & 94 & 68 & 102 & 99 \\
\hline Cs & ICPMS & $\mathrm{mg} / \mathrm{kg}$ & 2.1 & 0.26 & 1.6 & 2.4 & 3.2 & 2.2 & 4.0 & 2.5 & 1.8 & 1.9 & 1.9 & 2.2 & 2.4 \\
\hline $\mathrm{Cu}$ & ICPMS & $\mathrm{mg} / \mathrm{kg}$ & 4400 & 2240 & 2450 & 1440 & 1310 & 294 & 2020 & 374 & 3690 & 41 & 498 & 121 & 487 \\
\hline Dy & ICPMS & $\mathrm{mg} / \mathrm{kg}$ & 0.82 & 0.22 & 0.91 & 0.75 & 1.2 & 3.6 & 3.2 & 3.9 & n.a. & n.a. & n.a. & n.a. & n.a. \\
\hline $\mathrm{Er}$ & ICPMS & $\mathrm{mg} / \mathrm{kg}$ & 0.45 & 0.14 & 0.45 & 0.34 & 0.56 & 2.1 & 1.6 & 2.4 & n.a. & n.a. & n.a. & n.a. & n.a. \\
\hline $\mathrm{Eu}$ & ICPMS & $\mathrm{mg} / \mathrm{kg}$ & 0.42 & 0.066 & 0.38 & 0.29 & 0.49 & 0.93 & 1.1 & 1.0 & n.a. & n.a. & n.a. & n.a. & n.a. \\
\hline $\mathrm{Ga}$ & ICPMS & $\mathrm{mg} / \mathrm{kg}$ & 12 & 9.7 & 12 & 11 & 15 & 13 & 17 & 14 & 16 & 14 & 9.8 & 16 & 15 \\
\hline Gd & ICPMS & $\mathrm{mg} / \mathrm{kg}$ & 0.98 & 0.21 & 1.32 & 0.87 & 1.7 & 3.2 & 3.7 & 3.6 & n.a. & n.a. & n.a. & n.a. & n.a. \\
\hline $\mathrm{Ge}$ & ICPMS & $\mathrm{mg} / \mathrm{kg}$ & 23 & 2.2 & 4.0 & 1.4 & 1.9 & 0.75 & 1.7 & 0.67 & n.a. & n.a. & n.a. & n.a. & n.a. \\
\hline $\mathrm{Hg}$ & CVAA & $\mathrm{mg} / \mathrm{kg}$ & n.a. & n.a. & n.a. & n.a. & n.a. & n.a. & n.a. & n.a. & 1.3 & 0.060 & 0.66 & 0.23 & 0.76 \\
\hline Ho & ICPMS & $\mathrm{mg} / \mathrm{kg}$ & 0.15 & 0.041 & 0.17 & 0.12 & 0.21 & 0.76 & 0.62 & 0.81 & n.a. & n.a. & n.a. & n.a. & n.a. \\
\hline In & ICPMS & $\mathrm{mg} / \mathrm{kg}$ & 0.35 & 0.12 & 0.15 & 0.13 & 0.18 & $<0.01$ & 0.33 & 0.10 & n.a. & n.a. & n.a. & n.a. & n.a. \\
\hline $\mathrm{La}$ & ICPMS & $\mathrm{mg} / \mathrm{kg}$ & 7.0 & 2.0 & 11 & 8.8 & 15 & 14 & 17 & 16 & 12 & 16 & 12 & 18 & 18 \\
\hline $\mathrm{Li}$ & ICPMS & $\mathrm{mg} / \mathrm{kg}$ & 20 & 5.3 & 14 & 16 & 31 & 33 & 73 & 34 & 43 & 51 & 31 & 55 & 50 \\
\hline $\mathrm{Mn}$ & ICPMS & $\mathrm{mg} / \mathrm{kg}$ & 106 & 64 & 34 & 8.5 & 161 & 554 & 375 & 543 & 230 & 502 & 359 & 589 & 523 \\
\hline Mo & ICPMS & $\mathrm{mg} / \mathrm{kg}$ & 88 & 26 & 13 & 11 & 18 & 9.3 & 12 & 20 & 31 & 4.4 & 5.3 & 3.4 & 5.7 \\
\hline $\mathrm{Nb}$ & ICPMS & $\mathrm{mg} / \mathrm{kg}$ & 3.1 & 1.5 & 3.8 & 3.5 & 4.7 & 5.5 & 7.8 & 6.0 & 6.3 & 9.0 & 6.6 & 11 & 10 \\
\hline $\mathrm{Nd}$ & ICPMS & $\mathrm{mg} / \mathrm{kg}$ & 6.1 & 1.7 & 9.3 & 6.9 & 12 & 14 & 17 & 15 & n.a. & n.a. & n.a. & n.a. & n.a. \\
\hline
\end{tabular}

ICPMS = Inductively coupled plasma-mass spectrometry; CVAA = Cold vapor-atomic absorption spectrometry; n.a. = not analyzed 
Table 4. (cont.)

\begin{tabular}{|c|c|c|c|c|c|c|c|c|c|c|c|c|c|c|c|}
\hline \multicolumn{3}{|c|}{ Sample numbers } & $\begin{array}{l}\text { 03Ellamar- } \\
\text { Pit } 5\end{array}$ & $\begin{array}{l}\text { 03Ellamar- } \\
\text { Pit } 4\end{array}$ & $\begin{array}{l}\text { 03Ellamar- } \\
\text { Pit } 2\end{array}$ & $\begin{array}{l}\text { 03Ellamar- } \\
\text { Pit } 1\end{array}$ & $\begin{array}{l}\text { 03Ellamar- } \\
\text { Pit } 3\end{array}$ & $\begin{array}{l}\text { PWS03- } \\
\text { SELL-4 }\end{array}$ & $\begin{array}{l}\text { PWS03S- } \\
3\end{array}$ & $\begin{array}{l}\text { PWS030S- } \\
102\end{array}$ & $\begin{array}{l}\text { 05PWSE- } \\
002 \mathrm{~S}\end{array}$ & $\begin{array}{l}\text { 05PWSE- } \\
008 \mathrm{~S}\end{array}$ & $\begin{array}{l}\text { 05PWSE- } \\
108 \mathrm{~S}\end{array}$ & $\begin{array}{l}\text { 05PWSE- } \\
110 \mathrm{R}\end{array}$ & $\begin{array}{l}\text { 05PWSE- } \\
111 \mathrm{~S}\end{array}$ \\
\hline \multicolumn{3}{|c|}{ Mine site } & Ellamar & Ellamar & Ellamar & Ellamar & Ellamar & Ellamar & Ellamar & Ellamar & Ellamar & Ellamar & Ellamar & Ellamar & Ellamar \\
\hline \multicolumn{3}{|c|}{ Location } & intertidal & intertidal & intertidal & intertidal & intertidal & offshore & $\begin{array}{l}\text { glory } \\
\text { hole }\end{array}$ & offshore & intertidal & offshore & offshore & offshore & offshore \\
\hline Element & Method & Units & & & & & & & & & & & & & \\
\hline $\mathrm{Ni}$ & ICPMS & $\mathrm{mg} / \mathrm{kg}$ & 12 & 3.6 & 6.0 & 1.0 & 7.8 & 32 & 28 & 27 & 11 & 26 & 21 & 34 & 31 \\
\hline $\mathrm{Pb}$ & ICPMS & $\mathrm{mg} / \mathrm{kg}$ & 1480 & 297 & 581 & 33 & 139 & 149 & 202 & 44 & 128 & 13 & 56 & 28 & 66 \\
\hline $\operatorname{Pr}$ & ICPMS & $\mathrm{mg} / \mathrm{kg}$ & 1.6 & 0.44 & 2.5 & 1.9 & 3.3 & 3.3 & 4.0 & 3.8 & n.a. & n.a. & n.a. & n.a. & n.a. \\
\hline $\mathrm{Rb}$ & ICPMS & $\mathrm{mg} / \mathrm{kg}$ & 28 & 6.9 & 44 & 51 & 61 & 38 & 62 & 41 & 46 & 46 & 36 & 56 & 52 \\
\hline $\operatorname{Re}$ & ICPMS & $\mathrm{mg} / \mathrm{kg}$ & 0.052 & 0.012 & $<0.01$ & $<0.01$ & $<0.01$ & 0.01 & $<0.01$ & 0.011 & n.a. & n.a. & n.a. & n.a. & n.a. \\
\hline $\mathrm{Sb}$ & ICPMS & $\mathrm{mg} / \mathrm{kg}$ & 157 & 15 & 14 & 2.2 & 5.6 & 1.5 & 4.5 & 1.0 & 9.4 & 0.44 & 1.2 & 0.88 & 1.8 \\
\hline $\mathrm{Sc}$ & ICPMS & $\mathrm{mg} / \mathrm{kg}$ & 6.3 & 1.9 & 8.2 & 8.8 & 12 & 13 & 14 & 14 & 10 & 16 & 11 & 17 & 17 \\
\hline $\mathrm{Se}$ & ICPMS & $\mathrm{mg} / \mathrm{kg}$ & 58 & 30 & 32 & 26 & 21 & 2.4 & 7.2 & 2.0 & n.a. & n.a. & n.a. & n.a. & n.a. \\
\hline $\mathrm{Sm}$ & ICPMS & $\mathrm{mg} / \mathrm{kg}$ & 1.3 & 0.35 & 1.8 & 1.3 & 2.3 & 3.2 & 3.7 & 3.5 & n.a. & n.a. & n.a. & n.a. & n.a. \\
\hline $\mathrm{Sn}$ & ICPMS & $\mathrm{mg} / \mathrm{kg}$ & 182 & 10 & 8.4 & 4.0 & 4.6 & 364 & 25 & 25 & n.a. & n.a. & n.a. & n.a. & n.a. \\
\hline $\mathrm{Sr}$ & ICPMS & $\mathrm{mg} / \mathrm{kg}$ & 77 & 32 & 75 & 128 & 91 & 354 & 164 & 261 & 119 & 180 & 275 & 216 & 227 \\
\hline $\mathrm{Tb}$ & ICPMS & $\mathrm{mg} / \mathrm{kg}$ & 0.15 & 0.036 & 0.18 & 0.12 & 0.23 & 0.54 & 0.56 & 0.59 & n.a. & n.a. & n.a. & n.a. & n.a. \\
\hline $\mathrm{Te}$ & ICPMS & $\mathrm{mg} / \mathrm{kg}$ & 4.5 & 3.3 & 2.7 & 1.6 & 1.7 & $<0.2$ & 0.72 & $<0.2$ & n.a. & n.a. & n.a. & n.a. & n.a. \\
\hline Th & ICPMS & $\mathrm{mg} / \mathrm{kg}$ & 2.4 & 1.2 & 1.9 & 1.4 & 4.2 & 2.8 & 5.0 & 3.2 & 3.3 & 4.4 & 3.0 & 5.2 & 5.0 \\
\hline $\mathrm{Tl}$ & ICPMS & $\mathrm{mg} / \mathrm{kg}$ & 28 & 5.8 & 1.8 & 6.4 & 2.6 & 0.56 & 0.86 & 0.59 & 1.9 & 0.54 & 0.52 & 0.62 & 0.81 \\
\hline $\mathrm{Tm}$ & ICPMS & $\mathrm{mg} / \mathrm{kg}$ & 0.070 & 0.026 & 0.069 & 0.059 & 0.092 & 0.32 & 0.22 & 0.33 & n.a. & n.a. & n.a. & n.a. & n.a. \\
\hline $\mathrm{U}$ & ICPMS & $\mathrm{mg} / \mathrm{kg}$ & 2.0 & 0.83 & 0.91 & 0.64 & 1.4 & 3.2 & 3.1 & 6.8 & 4.5 & 2.8 & 2.2 & 2.3 & 3.3 \\
\hline V & ICPMS & $\mathrm{mg} / \mathrm{kg}$ & 95 & 43 & 77 & 91 & 150 & 154 & 172 & 146 & 136 & 134 & 144 & 161 & 164 \\
\hline $\mathrm{W}$ & ICPMS & $\mathrm{mg} / \mathrm{kg}$ & 4.2 & 0.66 & 0.81 & 0.55 & 0.81 & 0.98 & 1.4 & 0.92 & n.a. & n.a. & n.a. & n.a. & n.a. \\
\hline $\mathrm{Y}$ & ICPMS & $\mathrm{mg} / \mathrm{kg}$ & 2.8 & 0.91 & 3.2 & 2.5 & 4.1 & 18 & 13 & 19 & 5.2 & 14 & 14 & 16 & 17 \\
\hline $\mathrm{Yb}$ & ICPMS & $\mathrm{mg} / \mathrm{kg}$ & 0.41 & 0.16 & 0.43 & 0.34 & 0.54 & 1.7 & 1.3 & 1.8 & n.a. & n.a. & n.a. & n.a. & n.a. \\
\hline $\mathrm{Zn}$ & ICPMS & $\mathrm{mg} / \mathrm{kg}$ & 512 & 356 & 861 & 37 & 105 & 198 & 982 & 158 & 527 & 86 & 154 & 114 & 260 \\
\hline
\end{tabular}


Table 4. (cont.)

\begin{tabular}{|c|c|c|c|c|c|c|c|c|c|c|c|c|c|c|c|}
\hline \multicolumn{3}{|c|}{ Sample numbers } & $\begin{array}{l}\text { 05PWSE- } \\
112 \mathrm{~S} \\
\end{array}$ & $\begin{array}{l}\text { 05PWSE- } \\
207 \mathrm{~S}\end{array}$ & $\begin{array}{l}\text { 05PWSE- } \\
208 \mathrm{~S}\end{array}$ & $\begin{array}{l}\text { 05PWST- } \\
115 \mathrm{~S} \\
\end{array}$ & $\begin{array}{l}\text { 05PWST- } \\
118 \mathrm{~S}\end{array}$ & $\begin{array}{l}\text { 05PWST- } \\
120 \mathrm{~S} \\
\end{array}$ & $\begin{array}{l}\text { 05PWST- } \\
304 S-1\end{array}$ & $\begin{array}{l}\text { 05PWST- } \\
\text { 305SM-1 }\end{array}$ & $\begin{array}{l}\text { 05PWST- } \\
\text { 306SM-2 }\end{array}$ & $\begin{array}{l}\text { 05PWST- } \\
\text { 308SM-1 }\end{array}$ & $\begin{array}{l}\text { 05PWSB- } \\
010 \mathrm{~S}\end{array}$ & $\begin{array}{l}\text { 05PWSB- } \\
101 \mathrm{~S}\end{array}$ & $\begin{array}{l}\text { 05PWSB- } \\
104 \mathrm{~S}\end{array}$ \\
\hline \multicolumn{3}{|c|}{ Mine site } & Ellamar & Ellamar & Ellamar & Threeman & Threeman & Threeman & Threeman & Threeman & Threeman & Threeman & Beatson & Beatson & Beatson \\
\hline \multicolumn{3}{|c|}{ Location } & offshore & intertidal & intertidal & offshore & intertidal & offshore & intertidal & intertidal & intertidal & intertidal & offshore & offshore & offshore \\
\hline $\mathrm{Al}$ & ICPMS & wt. $\%$ & 6.88 & 4.69 & 1.71 & 8.52 & 5.08 & 8.2 & 10.4 & 10 & 7.14 & 1.36 & n.a. & n.a. & 7.18 \\
\hline $\mathrm{Ca}$ & ICPMS & wt. \% & 1.25 & 0.085 & 0.030 & 1.44 & 3.45 & 1.55 & 1.11 & 3.44 & 1.24 & 0.246 & 2.1 & 0.437 & 0.371 \\
\hline $\mathrm{Fe}$ & ICPMS & wt. \% & 3.6 & 18 & 34 & 4.0 & 18 & 4.3 & 4.8 & 9.9 & 7.6 & 47 & 5.8 & 5.1 & 6.1 \\
\hline $\mathrm{K}$ & ICPMS & wt. $\%$ & 1.66 & 1.48 & 0.538 & 1.54 & 0.597 & 1.57 & 1.94 & 0.71 & 0.284 & 0.0823 & 1.36 & 1.75 & 1.54 \\
\hline $\mathrm{Mg}$ & ICPMS & wt. \% & 1.51 & 0.538 & 0.197 & 1.78 & 2.01 & 2.06 & 2.15 & 4.66 & 3.50 & 0.654 & 2.84 & 1.8 & 2.26 \\
\hline $\mathrm{Na}$ & ICPMS & wt. \% & 1.50 & 0.658 & 0.249 & 2.93 & 0.987 & 3.10 & 2.33 & 1.43 & 1.98 & 0.130 & 1.24 & 1.49 & 1.12 \\
\hline $\mathrm{P}$ & ICPMS & wt. \% & 0.070 & 0.039 & 0.018 & 0.13 & 0.30 & 0.14 & 0.10 & 0.072 & 0.16 & 0.035 & 0.072 & 0.088 & 0.085 \\
\hline $\mathrm{Ti}$ & ICPMS & wt. \% & 0.43 & 0.20 & 0.081 & 0.060 & 0.28 & 0.50 & 0.50 & 0.55 & 0.32 & 0.049 & 0.25 & 0.28 & 0.22 \\
\hline $\mathrm{Ag}$ & ICPMS & $\mathrm{mg} / \mathrm{kg}$ & $<3$ & 7.9 & 12 & $<3$ & $<3$ & $<3$ & $<3$ & $<3$ & $<3$ & 3.3 & $<3$ & $<3$ & $<3$ \\
\hline As & ICPMS & $\mathrm{mg} / \mathrm{kg}$ & 11 & 170 & 380 & 9 & 67 & 11 & 9.1 & 7.9 & 9.7 & 21 & 38 & 24 & 50 \\
\hline $\mathrm{Au}$ & ICPMS & $\mathrm{mg} / \mathrm{kg}$ & n.a. & n.a. & n.a. & n.a. & n.a. & n.a. & n.a. & n.a. & n.a. & n.a. & n.a. & n.a. & n.a. \\
\hline $\mathrm{Ba}$ & ICPMS & $\mathrm{mg} / \mathrm{kg}$ & 801 & 183 & 223 & 660 & 274 & 643 & 909 & 299 & 93 & 21 & 760 & 923 & 838 \\
\hline $\mathrm{Be}$ & ICPMS & $\mathrm{mg} / \mathrm{kg}$ & 1.2 & 0.90 & 0.40 & 1.5 & 0.59 & 1.4 & 1.4 & 0.73 & 0.42 & 0.030 & 1.2 & 1.5 & 1.4 \\
\hline $\mathrm{Cd}$ & ICPMS & $\mathrm{mg} / \mathrm{kg}$ & 0.22 & 1.3 & 4.4 & 0.55 & 0.38 & 1.3 & 0.050 & 0.16 & 1.4 & 2.3 & 1.4 & 0.080 & 0.12 \\
\hline $\mathrm{Ce}$ & ICPMS & $\mathrm{mg} / \mathrm{kg}$ & 34 & 24 & 8.1 & 48 & 17 & 38 & 43 & 20 & 10 & 1.4 & 32 & 40 & 38 \\
\hline Co & ICPMS & $\mathrm{mg} / \mathrm{kg}$ & 7.9 & 38 & 187 & 10 & 12 & 13 & 14 & 27 & 28 & 204 & 19 & 11 & 16 \\
\hline $\mathrm{Cr}$ & ICPMS & $\mathrm{mg} / \mathrm{kg}$ & 103 & 57 & 24 & 89 & 98 & 94 & 105 & 280 & 192 & 43 & 94 & 105 & 92 \\
\hline Cs & ICPMS & $\mathrm{mg} / \mathrm{kg}$ & 2.1 & 2.0 & 0.73 & 3.2 & 1.2 & 3.3 & 2.9 & 0.99 & 0.49 & 0.080 & 2.7 & 3.5 & 3.2 \\
\hline $\mathrm{Cu}$ & ICPMS & $\mathrm{mg} / \mathrm{kg}$ & 60.5 & 2120 & 3090 & 88.6 & 1300 & 114 & 377 & 870 & 4690 & 25800 & 1590 & 1010 & 2580 \\
\hline Dy & ICPMS & $\mathrm{mg} / \mathrm{kg}$ & n.a. & n.a. & n.a. & n.a. & n.a. & n.a. & n.a. & n.a. & n.a. & n.a. & n.a. & n.a. & n.a. \\
\hline $\mathrm{Er}$ & ICPMS & $\mathrm{mg} / \mathrm{kg}$ & n.a. & n.a. & n.a. & n.a. & n.a. & n.a. & n.a. & n.a. & n.a. & n.a. & n.a. & n.a. & n.a. \\
\hline $\mathrm{Eu}$ & ICPMS & $\mathrm{mg} / \mathrm{kg}$ & n.a. & n.a. & n.a. & n.a. & n.a. & n.a. & n.a. & n.a. & n.a. & n.a. & n.a. & n.a. & n.a. \\
\hline $\mathrm{Ga}$ & ICPMS & $\mathrm{mg} / \mathrm{kg}$ & 16 & 14 & 7.1 & 15 & 8.9 & 15 & 17 & 16 & 9.2 & 2.7 & 18 & 17 & 18 \\
\hline Gd & ICPMS & $\mathrm{mg} / \mathrm{kg}$ & n.a. & n.a. & n.a. & n.a. & n.a. & n.a. & n.a. & n.a. & n.a. & n.a. & n.a. & n.a. & n.a. \\
\hline $\mathrm{Ge}$ & ICPMS & $\mathrm{mg} / \mathrm{kg}$ & n.a. & n.a. & n.a. & n.a. & n.a. & n.a. & n.a. & n.a. & n.a. & n.a. & n.a. & n.a. & n.a. \\
\hline $\mathrm{Hg}$ & CVAA & $\mathrm{mg} / \mathrm{kg}$ & 0.13 & 2.6 & 3.2 & 0.05 & 0.14 & 0.04 & 0.03 & 0.02 & 0.11 & $<0.02$ & 0.19 & 0.12 & 0.16 \\
\hline Ho & ICPMS & $\mathrm{mg} / \mathrm{kg}$ & n.a. & n.a. & n.a. & n.a. & n.a. & n.a. & n.a. & n.a. & n.a. & n.a. & n.a. & n.a. & n.a. \\
\hline In & ICPMS & $\mathrm{mg} / \mathrm{kg}$ & n.a. & n.a. & n.a. & n.a. & n.a. & n.a. & n.a. & n.a. & n.a. & n.a. & n.a. & n.a. & n.a. \\
\hline $\mathrm{La}$ & ICPMS & $\mathrm{mg} / \mathrm{kg}$ & 19 & 12 & 4.3 & 23 & 8.5 & 18 & 21 & 9.1 & 4.6 & 0.60 & 18 & 22 & 21 \\
\hline $\mathrm{Li}$ & ICPMS & $\mathrm{mg} / \mathrm{kg}$ & 61 & 44 & 17 & 52 & 24 & 55 & 60 & 37 & 21 & 2.8 & 46 & 56 & 52 \\
\hline $\mathrm{Nd}$ & ICPMS & $\mathrm{mg} / \mathrm{kg}$ & n.a. & n.a. & n.a. & n.a. & n.a. & n.a. & n.a. & n.a. & n.a. & n.a. & n.a. & n.a. & n.a. \\
\hline
\end{tabular}


Table 4. (cont.)

\begin{tabular}{|c|c|c|c|c|c|c|c|c|c|c|c|c|c|c|c|}
\hline \multicolumn{3}{|c|}{ Sample numbers } & $\begin{array}{l}\text { 05PWSE- } \\
112 \mathrm{~S}\end{array}$ & $\begin{array}{l}\text { 05PWSE- } \\
207 \mathrm{~S}\end{array}$ & $\begin{array}{l}\text { 05PWSE- } \\
208 \mathrm{~S}\end{array}$ & $\begin{array}{l}\text { 05PWST- } \\
115 \mathrm{~S}\end{array}$ & $\begin{array}{l}\text { 05PWST- } \\
118 \mathrm{~S}\end{array}$ & $\begin{array}{l}\text { 05PWST- } \\
120 \mathrm{~S}\end{array}$ & $\begin{array}{l}\text { 05PWST- } \\
304 \mathrm{~S}-1\end{array}$ & $\begin{array}{l}\text { 05PWST- } \\
\text { 305SM-1 }\end{array}$ & $\begin{array}{l}\text { 05PWST- } \\
\text { 306SM-2 }\end{array}$ & $\begin{array}{l}\text { 05PWST- } \\
\text { 308SM-1 }\end{array}$ & $\begin{array}{l}\text { 05PWSB- } \\
010 \mathrm{~S}\end{array}$ & $\begin{array}{l}\text { 05PWSB- } \\
101 \mathrm{~S}\end{array}$ & $\begin{array}{l}\text { 05PWSB- } \\
104 \mathrm{~S}\end{array}$ \\
\hline \multicolumn{3}{|c|}{ Mine site } & Ellamar & Ellamar & Ellamar & Threeman & Threeman & Threeman & Threeman & Threeman & Threeman & Threeman & Beatson & Beatson & Beatson \\
\hline \multicolumn{3}{|c|}{ Location } & offshore & intertidal & intertidal & offshore & intertidal & offshore & intertidal & intertidal & intertidal & intertidal & offshore & offshore & offshore \\
\hline Element & Method & Units & & & & & & & & & & & & & \\
\hline $\mathrm{Ni}$ & ICPMS & $\mathrm{mg} / \mathrm{kg}$ & 27 & 16 & 7.9 & 29 & 25 & 35 & 31 & 65 & 40 & 36 & 26 & 29 & 25 \\
\hline $\mathrm{Pb}$ & ICPMS & $\mathrm{mg} / \mathrm{kg}$ & 16 & 143 & 116 & 15 & 30 & 17 & 24 & 22 & 7.8 & 15 & 63 & 59 & 98 \\
\hline $\operatorname{Pr}$ & ICPMS & $\mathrm{mg} / \mathrm{kg}$ & n.a. & n.a. & n.a. & n.a. & n.a. & n.a. & n.a. & n.a. & n.a. & n.a. & n.a. & n.a. & n.a. \\
\hline $\mathrm{Rb}$ & ICPMS & $\mathrm{mg} / \mathrm{kg}$ & 57 & 46 & 18 & 55 & 20 & 55 & 66 & 22 & 5.8 & 1.6 & 54 & 70 & 61 \\
\hline $\operatorname{Re}$ & ICPMS & $\mathrm{mg} / \mathrm{kg}$ & n.a. & n.a. & n.a. & n.a. & n.a. & n.a. & n.a. & n.a. & n.a. & n.a. & n.a. & n.a. & n.a. \\
\hline $\mathrm{Sb}$ & ICPMS & $\mathrm{mg} / \mathrm{kg}$ & 0.48 & 14 & 15 & 0.62 & 0.82 & 0.82 & 0.86 & 0.47 & 0.35 & 0.90 & 1.1 & 1.4 & 2.9 \\
\hline $\mathrm{Sc}$ & ICPMS & $\mathrm{mg} / \mathrm{kg}$ & 17 & 9.3 & 3.6 & 16 & 14 & 18 & 20 & 34 & 23 & 4.4 & 15 & 16 & 15 \\
\hline $\mathrm{Se}$ & ICPMS & $\mathrm{mg} / \mathrm{kg}$ & n.a. & n.a. & n.a. & n.a. & n.a. & n.a. & n.a. & n.a. & n.a. & n.a. & n.a. & n.a. & n.a. \\
\hline $\mathrm{Sm}$ & ICPMS & $\mathrm{mg} / \mathrm{kg}$ & n.a. & n.a. & n.a. & n.a. & n.a. & n.a. & n.a. & n.a. & n.a. & n.a. & n.a. & n.a. & n.a. \\
\hline $\mathrm{Sn}$ & ICPMS & $\mathrm{mg} / \mathrm{kg}$ & n.a. & n.a. & n.a. & n.a. & n.a. & n.a. & n.a. & n.a. & n.a. & n.a. & n.a. & n.a. & n.a. \\
\hline $\mathrm{Sr}$ & ICPMS & $\mathrm{mg} / \mathrm{kg}$ & 200 & 87 & 33 & 246 & 365 & 233 & 222 & 210 & 135 & 15 & 238 & 181 & 144 \\
\hline $\mathrm{Tb}$ & ICPMS & $\mathrm{mg} / \mathrm{kg}$ & n.a. & n.a. & n.a. & n.a. & n.a. & n.a. & n.a. & n.a. & n.a. & n.a. & n.a. & n.a. & n.a. \\
\hline $\mathrm{Te}$ & ICPMS & $\mathrm{mg} / \mathrm{kg}$ & n.a. & n.a. & n.a. & n.a. & n.a. & n.a. & n.a. & n.a. & n.a. & n.a. & n.a. & n.a. & n.a. \\
\hline $\mathrm{Tl}$ & ICPMS & $\mathrm{mg} / \mathrm{kg}$ & 0.56 & 4.0 & 8.0 & 0.50 & 0.20 & 0.55 & 0.40 & 0.20 & 0.080 & 0.020 & 0.50 & 0.55 & 0.51 \\
\hline $\mathrm{Tm}$ & ICPMS & $\mathrm{mg} / \mathrm{kg}$ & n.a. & n.a. & n.a. & n.a. & n.a. & n.a. & n.a. & n.a. & n.a. & n.a. & n.a. & n.a. & n.a. \\
\hline $\mathrm{U}$ & ICPMS & $\mathrm{mg} / \mathrm{kg}$ & 2.6 & 2.5 & 1.1 & 3.6 & 2.6 & 8.6 & 2.4 & 1.5 & 6.5 & 7.3 & 1.5 & 1.5 & 1.7 \\
\hline V & ICPMS & $\mathrm{mg} / \mathrm{kg}$ & 155 & 114 & 47 & 134 & 172 & 151 & 177 & 258 & 140 & 42 & 137 & 163 & 159 \\
\hline W & ICPMS & $\mathrm{mg} / \mathrm{kg}$ & n.a. & n.a. & n.a. & n.a. & n.a. & n.a. & n.a. & n.a. & n.a. & n.a. & n.a. & n.a. & n.a. \\
\hline Y & ICPMS & $\mathrm{mg} / \mathrm{kg}$ & 15 & 6.7 & 2.2 & 21 & 14 & 20 & 15 & 19 & 15 & 2.2 & 14 & 18 & 16 \\
\hline $\mathrm{Yb}$ & ICPMS & $\mathrm{mg} / \mathrm{kg}$ & n.a. & n.a. & n.a. & n.a. & n.a. & n.a. & n.a. & n.a. & n.a. & n.a. & n.a. & n.a. & n.a. \\
\hline $\mathrm{Zn}$ & ICPMS & $\mathrm{mg} / \mathrm{kg}$ & 87 & 854 & 820 & 113 & 260 & 132 & 139 & 289 & 938 & 1100 & 684 & 233 & 350 \\
\hline
\end{tabular}




\title{
Chapter D. Chemical Data for Biological Samples
}

\author{
By LeeAnn Munk and Randolph A. Koski
}

Field investigations of sulfide oxidation at abandoned mine sites in coastal areas of Prince William Sound were conducted in 2003 and 2005. In 2005 marine organisms including mussels, barnacles, gastropods, clams, limpets, eelgrass, and algae were collected from intertidal zones at the Ellamar, Threeman, and Beatson sites (table 5). Biological samples were frozen shortly after collection, and were analyzed in laboratories of the U.S. Geological Survey in Denver, Colorado (table 6). Tissue from animal specimens was hand picked from shell material using tweezers and then freeze dried prior to grinding and analysis. Major and trace elements were determined by inductively coupled plasma-mass spectrometry following multi-acid ( $\mathrm{HCl}-\mathrm{HNO}_{3}-\mathrm{HClO}_{4}-\mathrm{HF}$ ) digestion (Briggs and Meier, 2002) except for mercury, which was analyzed by cold-vapor atomic absorption spectroscopy (Brown et al., 2002). Details regarding sample preparation and detection limits are found in Taggert (2002). Base metal concentrations in mussels collected near the three mine sites are compared to similar data for mussels from other locations in Prince William Sound in Koski and others (2008).

Table 5. Types and locations of biological samples.

\begin{tabular}{llllll}
\hline Sample number & $\begin{array}{l}\text { Sample } \\
\text { type }\end{array}$ & Mine site & Latitude & Longitude & Location description \\
\hline 05PWS-E201m & mussel & Ellamar & $60^{\circ} 53.653^{\prime}$ & $-146^{\circ} 42.093^{\prime}$ & old pier piling \\
05PWS-T2 & mussel & Threeman & $60^{\circ} 51.180^{\prime}$ & $-146^{\circ} 32.257^{\prime}$ & rocks near mine debris \\
05PWS-T & mussel & Threeman & $60^{\circ} 51.206^{\prime}$ & $-146^{\circ} 32.228^{\prime}$ & rocks away from mine debris \\
05PWS-204B & mussel & Beatson & $60^{\circ} 03.145^{\prime}$ & $-146^{\circ} 54.436^{\prime}$ & rocky point west of mine debris \\
05PWS-204Bm & mussel & Beatson & $60^{\circ} 03.145^{\prime}$ & $-146^{\circ} 54.436^{\prime}$ & rocky point west of mine debris \\
05PWS-203Bm & mussel & Beatson & $60^{\circ} 03.145^{\prime}$ & $-146^{\circ} 54.436^{\prime}$ & rocky point west of mine debris \\
05PWS-T1b & barnacle & Threeman & $60^{\circ} 51.180^{\prime}$ & $-146^{\circ} 32.257^{\prime}$ & rocks near mine debris \\
05PWS-204BA & barnacle & Beatson & $60^{\circ} 03.145^{\prime}$ & $-146^{\circ} 54.436^{\prime}$ & rocky point west of mine debris \\
05PWS-204BB & barnacle & Beatson & $60^{\circ} 03.145^{\prime}$ & $-146^{\circ} 54.436^{\prime}$ & rocky point west of mine debris \\
05PWS-T1g & gastropod & Threeman & $60^{\circ} 51.180^{\prime}$ & $-146^{\circ} 32.257^{\prime}$ & rocks near mine debris \\
05PWS-203Bs & gastropod & Beatson & $60^{\circ} 03.145^{\prime}$ & $-146^{\circ} 54.436^{\prime}$ & rocky point west of mine debris \\
05PWS-T201B & clam & Threeman & $60^{\circ} 51.206^{\prime}$ & $-146^{\circ} 32.228^{\prime}$ & beach sand near mine debris \\
05PWS-T11 & limpet & Threeman & $60^{\circ} 51.180^{\prime}$ & $-146^{\circ} 32.257^{\prime}$ & rocks near mine debris \\
05PWS-E201e & eelgrass & Ellamar & $60^{\circ} 53.653^{\prime}$ & $-146^{\circ} 42.093^{\prime}$ & shallow offshore near mine debris \\
05PWS-E201a & algae & Ellamar & $60^{\circ} 53.653^{\prime}$ & $-146^{\circ} 42.093^{\prime}$ & intertidal algal mat near mine debris \\
\hline
\end{tabular}


Table 6. Chemical data (dry weight) for biological samples.

\begin{tabular}{|c|c|c|c|c|c|c|c|c|c|c|c|c|c|c|c|c|c|}
\hline \multicolumn{3}{|c|}{ Sample numbers } & $\begin{array}{l}\text { 05PWS- } \\
\text { E201m }\end{array}$ & $\begin{array}{l}\text { 05PWS- } \\
\text { T2 }\end{array}$ & 05PWS-T & $\begin{array}{l}\text { 05PWS- } \\
\text { 204B }\end{array}$ & $\begin{array}{l}\text { 05PWS- } \\
\text { 204Bm }\end{array}$ & $\begin{array}{l}\text { 05PWS- } \\
\text { 203Bm }\end{array}$ & $\begin{array}{l}\text { 05PWS- } \\
\text { T1b }\end{array}$ & $\begin{array}{l}\text { 05PWS- } \\
\text { 204BA }\end{array}$ & $\begin{array}{l}\text { 05PWS- } \\
\text { 204BB }\end{array}$ & $\begin{array}{l}\text { 05PWS- } \\
\text { T1g }\end{array}$ & $\begin{array}{l}\text { 05PWS- } \\
\text { 203Bs }\end{array}$ & $\begin{array}{l}\text { 05PWS- } \\
\text { T201B }\end{array}$ & $\begin{array}{l}\text { 05PWS- } \\
\text { T11 }\end{array}$ & $\begin{array}{l}\text { 05PWS- } \\
\text { E201e }\end{array}$ & $\begin{array}{l}\text { 05PWS- } \\
\text { E201a }\end{array}$ \\
\hline \multicolumn{3}{|c|}{ Organism } & mussel & mussel & mussel & mussel & mussel & mussel & barnacle & barnacle & barnacle & gastropod & gastropod & clam & limpet & eelgrass & algae \\
\hline \multicolumn{3}{|c|}{ Mine site } & Ellamar & Threeman & Threeman & Beatson & Beatson & Beatson & Threeman & Beatson & Beatson & Threeman & Beatson & Threeman & Threeman & Ellamar & Ellamar \\
\hline Element & Method & Units & & & & & & & & & & & & & & & \\
\hline $\mathrm{Al}$ & ICPMS & wt. \% & 0.050 & 0.036 & 0.046 & 0.042 & 0.22 & 0.050 & 0.055 & 0.34 & 0.14 & 0.14 & 0.11 & 0.037 & 0.34 & 0.12 & 1.74 \\
\hline $\mathrm{Ca}$ & ICPMS & wt. \% & 1.20 & 0.86 & 1.05 & 0.59 & 1.05 & 0.80 & 3.56 & 2.86 & 10.5 & 0.55 & 0.64 & 1.12 & 2.46 & 1.46 & 1.61 \\
\hline $\mathrm{Fe}$ & ICPMS & wt. \% & 0.037 & 0.036 & 0.026 & 0.041 & 0.098 & 0.05 & 0.081 & 0.20 & 0.080 & 0.12 & 0.099 & 0.036 & 0.36 & 0.70 & 4.0 \\
\hline K & ICPMS & wt. \% & 0.71 & 0.68 & 0.89 & 0.63 & 0.95 & 0.57 & 0.31 & 0.76 & 0.53 & 0.93 & 0.76 & 0.93 & 0.68 & 0.55 & 0.45 \\
\hline $\mathrm{Mg}$ & ICPMS & wt. \% & 0.59 & 0.40 & 0.70 & 0.27 & 0.54 & 0.29 & 0.53 & 0.93 & 0.77 & 1.45 & 0.83 & 0.70 & 0.53 & 1.64 & 1.32 \\
\hline $\mathrm{Na}$ & ICPMS & wt. $\%$ & 3.23 & 2.04 & 4.34 & 1.22 & 2.58 & 1.16 & 1.97 & 4.42 & 3.27 & 1.50 & 1.17 & 4.33 & 2.26 & 1.87 & 0.76 \\
\hline $\mathrm{P}$ & ICPMS & wt. \% & 1.3 & 0.92 & 1.2 & 1.3 & 2.0 & 1.2 & 0.61 & 1.2 & 0.80 & 1.0 & 0.86 & 1.1 & 1.0 & 0.32 & 0.19 \\
\hline $\mathrm{Ti}$ & ICPMS & wt. \% & $<0.004$ & $<0.004$ & $<0.004$ & $<0.004$ & 0.0051 & $<0.004$ & $<0.004$ & 0.011 & $<0.004$ & 0.0069 & 0.0042 & $<0.004$ & 0.016 & 0.0046 & 0.059 \\
\hline $\mathrm{Ag}$ & ICPMS & $\mathrm{mg} / \mathrm{kg}$ & $<3$ & $<3$ & $<3$ & $<3$ & $<3$ & $<3$ & $<3$ & $<3$ & $<3$ & $<3$ & $<3$ & $<3$ & $<3$ & $<3$ & $<3$ \\
\hline As & ICPMS & $\mathrm{mg} / \mathrm{kg}$ & 5.8 & 9.7 & 5.7 & 7.0 & 5.6 & 6.9 & 9.4 & 8.0 & 6.6 & 12 & 12 & 13 & 10 & 7.3 & 39 \\
\hline $\mathrm{Ba}$ & ICPMS & $\mathrm{mg} / \mathrm{kg}$ & 4.8 & 3.5 & 5.4 & 9.9 & 21 & 13 & 18 & 86 & 81 & 7.7 & 19 & 5.9 & 22 & 12 & 159 \\
\hline $\mathrm{Be}$ & ICPMS & $\mathrm{mg} / \mathrm{kg}$ & 0.008 & 0.007 & 0.004 & 0.005 & 0.03 & 0.02 & 0.01 & 0.04 & 0.003 & 0.01 & 0.04 & 0.007 & 0.05 & 0.01 & 0.18 \\
\hline $\mathrm{Bi}$ & ICPMS & $\mathrm{mg} / \mathrm{kg}$ & $<0.005$ & 0.008 & $<0.005$ & 0.005 & 0.010 & 0.009 & 0.030 & 0.020 & 0.030 & 0.020 & 0.020 & $<0.005$ & 0.020 & 0.040 & 0.37 \\
\hline $\mathrm{Cd}$ & ICPMS & $\mathrm{mg} / \mathrm{kg}$ & 3.0 & 5.9 & 1.0 & 3.4 & 3.6 & 3.2 & 13 & 6.8 & 4.0 & 0.95 & 1.3 & 1.5 & 4.6 & 0.69 & 0.14 \\
\hline $\mathrm{Ce}$ & ICPMS & $\mathrm{mg} / \mathrm{kg}$ & $<0.5$ & $<0.5$ & $<0.5$ & $<0.5$ & $<0.5$ & $<0.5$ & $<0.5$ & 0.97 & 18.7 & 0.5 & 0.78 & $<0.5$ & 1.2 & 0.58 & 5.4 \\
\hline Co & ICPMS & $\mathrm{mg} / \mathrm{kg}$ & 0.79 & 1.1 & 1.3 & 0.68 & 0.86 & 0.81 & 2.0 & 1.5 & 0.89 & 2.9 & 3.7 & 1.1 & 2.8 & 1.3 & 3.6 \\
\hline $\mathrm{Cr}$ & ICPMS & $\mathrm{mg} / \mathrm{kg}$ & 2.0 & 2.6 & 1.8 & 2.9 & 3.3 & 2.7 & 8.8 & 4.9 & 12 & 3.8 & 3.6 & 2.2 & 8.2 & 3.2 & 30 \\
\hline Cs & ICPMS & $\mathrm{mg} / \mathrm{kg}$ & 0.02 & 0.02 & 0.02 & 0.04 & 0.08 & 0.04 & 0.03 & 0.11 & 0.04 & 0.04 & 0.08 & 0.02 & 0.10 & 0.04 & 0.44 \\
\hline $\mathrm{Cu}$ & ICPMS & $\mathrm{mg} / \mathrm{kg}$ & 45 & 74 & 68 & 86 & 67 & 58 & 564 & 451 & 239 & 1320 & 1020 & 227 & 304 & 157 & 1200 \\
\hline Ga & ICPMS & $\mathrm{mg} / \mathrm{kg}$ & $<0.006$ & 0.10 & $<0.006$ & 0.20 & 0.20 & 0.20 & 0.20 & 0.34 & 0.02 & 0.07 & 0.34 & $<0.006$ & 0.37 & $<0.006$ & 3.2 \\
\hline $\mathrm{Hg}$ & CVAA & $\mathrm{mg} / \mathrm{kg}$ & 0.07 & 0.07 & 0.03 & 0.04 & 0.08 & 0.08 & n.a. & 0.08 & n.a. & 0.11 & 0.07 & 0.03 & 0.07 & 0.04 & 0.67 \\
\hline $\mathrm{La}$ & ICPMS & $\mathrm{mg} / \mathrm{kg}$ & $<0.3$ & $<0.3$ & $<0.3$ & $<0.3$ & $<0.3$ & $<0.3$ & $<0.3$ & 0.50 & 1.9 & 0.32 & 0.48 & $<0.3$ & 0.71 & 0.32 & 2.6 \\
\hline $\mathrm{Li}$ & ICPMS & $\mathrm{mg} / \mathrm{kg}$ & 1.6 & 1.8 & 1.0 & 1.8 & 2.4 & 1.8 & 4.2 & 3.4 & 2.4 & 0.80 & 1.8 & 0.30 & 1.6 & 0.90 & 9 \\
\hline $\mathrm{Mn}$ & ICPMS & $\mathrm{mg} / \mathrm{kg}$ & 9.7 & 8.3 & 17 & 8 & 14 & 11 & 13 & 26 & 27 & 50 & 49 & 13 & 50 & 49 & 58 \\
\hline Mo & ICPMS & $\mathrm{mg} / \mathrm{kg}$ & 0.27 & 0.41 & 0.53 & 0.26 & 0.26 & 0.30 & 0.60 & 0.59 & 0.77 & 0.46 & 0.47 & 0.46 & 0.38 & 12 & 7.2 \\
\hline $\mathrm{Nb}$ & ICPMS & $\mathrm{mg} / \mathrm{kg}$ & $<2$ & $<2$ & $<2$ & $<2$ & $<2$ & $<2$ & $<2$ & $<2$ & $<2$ & $<2$ & $<2$ & $<2$ & $<2$ & $<2$ & $<2$ \\
\hline $\mathrm{Ni}$ & ICPMS & $\mathrm{mg} / \mathrm{kg}$ & 1.0 & 1.4 & 1.1 & $<1$ & 1.2 & 1.2 & 5.4 & 2.4 & 5.3 & 6.3 & 8.1 & 2.2 & 11 & 2.0 & 3.9 \\
\hline $\mathrm{Pb}$ & ICPMS & $\mathrm{mg} / \mathrm{kg}$ & 0.95 & 0.92 & 0.30 & 2.4 & 3.0 & 2.9 & 1.5 & 3.4 & 3.9 & 1.9 & 3.5 & $<0.2$ & 4.2 & 4.9 & 35 \\
\hline $\mathrm{Rb}$ & ICPMS & $\mathrm{mg} / \mathrm{kg}$ & 2.7 & 3.2 & 2.9 & 3.8 & 4.4 & 3.6 & 1.6 & 4.4 & 2.6 & 3.8 & 5.2 & 3.2 & 3.6 & 2.0 & 9.6 \\
\hline $\mathrm{Sb}$ & ICPMS & $\mathrm{mg} / \mathrm{kg}$ & $<0.02$ & $<0.02$ & $<0.02$ & $<0.02$ & $<0.02$ & 0.89 & 0.03 & 0.04 & 0.20 & $<0.02$ & 0.04 & $<0.02$ & 0.04 & 0.31 & 1.7 \\
\hline $\mathrm{Sc}$ & ICPMS & $\mathrm{mg} / \mathrm{kg}$ & $<0.3$ & $<0.3$ & $<0.3$ & $<0.3$ & $<0.3$ & $<0.3$ & 0.4 & 0.5 & $<0.3$ & $<0.3$ & 0.4 & $<0.3$ & 0.6 & $<0.3$ & 2.8 \\
\hline $\mathrm{Sr}$ & ICPMS & $\mathrm{mg} / \mathrm{kg}$ & 85 & 82 & 87 & 53 & 81 & 55 & 328 & 243 & 799 & 60 & 73 & 86 & 187 & 271 & 378 \\
\hline Th & ICPMS & $\mathrm{mg} / \mathrm{kg}$ & $<0.03$ & $<0.03$ & $<0.03$ & 0.03 & 0.08 & 0.03 & 0.05 & 0.16 & 0.07 & 0.08 & 0.09 & $<0.03$ & 0.20 & 0.08 & 0.85 \\
\hline $\mathrm{Tl}$ & ICPMS & $\mathrm{mg} / \mathrm{kg}$ & 0.01 & 0.01 & 0.007 & 0.01 & 0.02 & 0.02 & 0.02 & 0.02 & 0.02 & 0.02 & 0.03 & 0.009 & 0.02 & 0.03 & 0.3 \\
\hline $\mathrm{U}$ & ICPMS & $\mathrm{mg} / \mathrm{kg}$ & 0.15 & 0.2 & 0.23 & 0.1 & 0.12 & 0.16 & 0.90 & 0.59 & 0.65 & 0.40 & 0.37 & 0.31 & 0.62 & 0.99 & 1.8 \\
\hline V & ICPMS & $\mathrm{mg} / \mathrm{kg}$ & 1.5 & 1.3 & 1.6 & 2.4 & 4.2 & 2.5 & 2.0 & 7.0 & 3.1 & 3.8 & 4.5 & 1.6 & 6.7 & 15 & 48 \\
\hline Y & ICPMS & $\mathrm{mg} / \mathrm{kg}$ & $<0.3$ & $<0.3$ & $<0.3$ & $<0.3$ & 0.32 & $<0.3$ & 0.39 & 0.73 & 0.71 & 0.47 & 0.49 & 0.30 & 1.1 & 0.66 & 2.5 \\
\hline $\mathrm{Zn}$ & ICPMS & $\mathrm{mg} / \mathrm{kg}$ & 117 & 117 & 157 & 177 & 174 & 304 & 890 & 990 & 610 & 135 & 117 & 91 & 150 & 100 & 209 \\
\hline
\end{tabular}

ICPMS = Inductively coupled plasma-mass spectrometry; CVAA = Cold vapor-atomic absorption spectrometry; n.a. = not analyzed 


\section{Acknowledgments}

We acknowledge Pamela Gemery of the USGS for her diligent efforts in processing samples and facilitating analytical work. Steve Nelson (USGS, retired) participated in the fieldwork and added substantial regional expertise to the project. Submarine observations and sampling were greatly facilitated through the use of an underwater camera system operated by Hank Chezar (USGS, Western Region Coastal and Marine Geology Team) and a remotely operated vehicle owned and operated by Dave Lovalvo (Eastern Oceanics, Inc.). We thank Kevin O’Toole, Mike Boyle, and Jerry O’Brien (USGS, Western Region Coastal and Marine Geology Team) for marine technical and logistical support prior to our field studies. We appreciate the superb support, friendship, and patience of David Janka, captain of the research vessel Auklet, and Annette Janka during all phases of our field studies in Prince William Sound. We also thank Chugach Alaska Corporation, represented by Mike Hoyt and Dave Phillips, and Tatitlek Corporation, represented by Clare Doig, for securing land use permits allowing access to corporation lands near the Ellamar and Threeman mine sites.

Financial support for this study was provided by the Mineral Resources Program and the Coastal and Marine Geology Program, U.S. Geological Survey; West Coast \& Polar Regions Undersea Research Center (part of NOAA's National Undersea Research Program), University of Alaska, Fairbanks; Marine Minerals Technology Center-Arctic Seas Division, University of Alaska, Fairbanks; and the University of Alaska, Anchorage.

This report has been significantly improved following the thoughtful reviews of Barry Moring and John Galloway.

\section{References Cited}

Briggs, P.H., and Meier, A.L., 2002, The determination of forty-two elements in geological materials by inductively coupled plasma-mass spectrometry, in Taggert, J.E., Jr., ed., Analytical methods for chemical analysis of geologic and other materials, U.S. Geological Survey: U.S. Geological Survey Open-File Report 02-0223-I, ver. 5 at

\section{http://pubs.usgs.gov/of/2002/ofr-02-0223/.}


Brown, Z.A., and Curry, K.J., 2002a, Total sulfur by combustion, in Taggert, J.E., Jr., ed., Analytical methods for chemical analysis of geologic and other materials, U.S. Geological Survey: U.S. Geological Survey Open-File Report 02-0223-Q, ver. 5 at http://pubs.usgs.gov/of/2002/ofr-02-0223/.

Brown, Z.A., and Curry, K.J., 2002b, Total carbon by combustion, in Taggert, J.E., Jr., ed., Analytical methods for chemical analysis of geologic and other materials, U.S. Geological Survey: U.S. Geological Survey Open-File Report 02-0223-R, ver. 5 at http://pubs.usgs.gov/of/2002/ofr-02-0223/.

Brown, Z.A., O’Leary, R.M., Hageman, P.L., and Crock, J.G., 2002a, Mercury in water, geologic, and plant materials by continuous flow-cold vapor-atomic absorption spectrometry, in Taggert, J.E., Jr., ed., Analytical methods for chemical analysis of geologic and other materials, U.S. Geological Survey: U.S. Geological Survey Open-File Report 02-0223-M, ver. 5 at http://pubs.usgs.gov/of/2002/ofr-02-0223/.

Brown, Z.A., Papp, Clara, Brandt, Elaine, and Aruscavage, Phillip, 2002b, Carbonate carbon by coulometric titration, in Taggert, J.E., Jr., ed., Analytical methods for chemical analysis of geologic and other materials, U.S. Geological Survey: U.S. Geological Survey Open-File Report 02-0223-S, ver. 5 at http://pubs.usgs.gov/of/2002/ofr-02-0223/.

Foster, A.L., Munk, LeeAnn, Koski, R.A., Shanks, W.C., III, and Stillings, L.L., 2008, Relationships between microbial communities and environmental parameters at sites impacted by mining of volcanogenic massive sulfide deposits, Prince William Sound, Alaska: Journal of Applied Geochemistry (in press).

Hageman, P.L., Brown, Z.A., and Welsch, Eric, 2002, Arsenic and selenium by flow injection or continuous flow-hydride generation-atomic absorption spectrophotometry, in Taggert, J.E., Jr., ed., Analytical methods for chemical analysis of geologic and other materials, U.S. Geological Survey: U.S. Geological Survey Open-File Report 02-0223-L, ver. 5 at http://pubs.usgs.gov/of/2002/ofr-02-0223/.

Koski, R.A., Munk, LeeAnn, Foster, A.L., Shanks, W.C., III, and Stillings, L.L., 2008, Sulfide oxidation and distribution of metals near abandoned copper mines in coastal environments, Prince William Sound, Alaska: Journal of Applied Geochemistry (in press). 
Stillings, L.L., Foster, A.L., Koski, R.A., Munk, LeeAnn, and Shanks, W.C., III, 2008, Temporal variation and the effect of rainfall on metals flux from the historic Beatson Mine, Prince William Sound, Alaska: Journal of Applied Geochemistry (in press).

Taggart, J.E., Jr., ed., 2002, Analytical methods for chemical analysis of geologic and other materials, U.S. Geological Survey: U.S. Geological Survey Open-File Report 02-0223, ver. 5 (http://pubs.usgs.gov/of/2002/ofr-02-0223/) 